Research Article

\title{
Posbist Reliability Theory for Typical Systems with Multicomponents
}

\author{
Jiaojiao Ren (iD) ${ }^{1}$ and Cong $\mathrm{Wu}^{2}$ \\ ${ }^{1}$ School of Information Science and Engineering, Chengdu University, Chengdu 610106, China \\ ${ }^{2}$ Institute of Nuclear Physics and Chemistry, China Academy of Engineering Physics, Mianyang 621900, China \\ Correspondence should be addressed to Jiaojiao Ren; jiaojiaoren06@163.com
}

Received 15 February 2020; Revised 15 April 2020; Accepted 12 May 2020; Published 4 June 2020

Academic Editor: Inmaculada T. Castro

Copyright (c) 2020 Jiaojiao Ren and Cong Wu. This is an open access article distributed under the Creative Commons Attribution License, which permits unrestricted use, distribution, and reproduction in any medium, provided the original work is properly cited.

\begin{abstract}
This paper investigates Posbist reliability theory for typical systems with multicomponents. This theory is based on possibility and binary-state assumptions. It works well under situations of insufficient data and epistemic uncertainty. When the lifetime of every component of a typical system is assumed unrelated and a normal, strictly convex fuzzy variable in some possibility space, the Posbist reliability is concretely derived where the exactly value can be obtained at any moment, unlike existing ones only given by a lower and upper bound. We numerically compare the Posbist reliability between typical systems of four types: series, parallel, series-parallel, and parallel-series. Moreover, numerical safety evaluations are given based on possibilistic and probabilistic models.
\end{abstract}

\section{Introduction}

Failures are always inevitable in many industrial systems. To measure the uncertainty of failures, the probabilistic reliability method, defining reliability by probability, is a remarkably successful tool [1-7]. As for the complex structure in practical engineering, natural language is widely used to express the fuzzy information. Another useful theory, the fuzzy reliability theory, was developed. This theory is more advantageous than the probabilistic reliability method, especially in situations that suffer lack of necessary sample data, subjective information, or epistemic uncertainty.

The fuzzy reliability theory is based on the fuzzy mathematics theory [8], and the possibility theory [9], employing fuzzy states and possibility rather than binary states and probability, was presented by Onisawa in [10]. Later, the theory was further developed to be three branches: the Posbist reliability theory based on possibility and binary states [11-13]; Profust reliability theory based on probability and fuzzy states [14-16]; and Posfust reliability theory based on possibility and fuzzy states [17]. Afterwards, a new possibilistic reliability index definition was proposed [18]. The fuzzy reliability theory was then extended to structural reliability analysis [19] and reliability analysis of system with fuzzy random variables [20]. In the recent past, a variant of the theory, based on chance measure, to assess mechanism reliability under fuzzy and random uncertainties, was published [21].

In the field of reliability theory, the Posbist reliablity theory has drawn plenty of attention because it is particularly reliable when applied to those systems about which only vague data, small samples, and spare statistical charateristics are availble. By adopting the traditional definition of reliability, Posbist reliability models of typical systems had been constructed in $[11,12]$. However, typical systems in $[11,12]$ were required to be irreparable. The authors in [13] established Posbist reliability models of repairable typical systems. Recently, the Posbist reliability theory was studied again in $[22,23]$. Furthermore, some perspectives to define and model reliability using fuzzy sets were given in [24]. However, in most existing results, the considered typical systems only investigated two components with different possibility distribution functions and $n$ components with the same possibility distribution functions. In [25], the possibility distribution function of the lifetime of a system with $n$ 
different components was obtained. However, there was only a lower bound and a upper bound, i.e., $\min _{1 \leq i \leq n}\left(\mu_{X_{i}}(x)\right) \leq \mu_{X}(x) \leq \max _{1 \leq i \leq n}\left(\mu_{X_{i}}(x)\right)$, without any concretely results. Furthermore, in [26], the authors considered typical systems with $n$ components where distribution functions of the lifetime of the components were assumed to be different. Moreover, the authors in [26] assumed the lifetime of each component as a symmetrical Gaussian fuzzy variable. Parameter $h_{i}$ in the distribution functions was assumed to be the same. Therefore, for more general cases, a given system has not only a symmetrical Gaussian fuzzy variable but also multiple components with different possibility distribution functions. Obtaining possibility distribution function of the system lifetime and Posbist reliability is a challenging problem.

Motivated by abovementioned discussions, due to the existence of insufficient and vague data in practical systems, the problem of Posbist reliability modeling and analysis for typical systems with multicomponents is further investigated in this paper via the possibility theory. The lifetime of components are assumed as unrelated and each a normal, strictly convex fuzzy variable, rather than a Gaussian fuzzy variable. The Posbist reliability theory of typical systems is concretely obtained where the exactly value can be obtained at any moment. Finally, numerical examples are provided to illustrate the feasibility of the obtained results.

\section{Preliminaries}

Based on Posbist reliability theory and the existence theorem of the possibility space, see pages 62-69 in [25], a possibility space $\left(U, \Phi, P_{\text {oss }}\right)$ is assumed to characterize the uncertainties of failure time of the system and its components by the context of possibility measures. Before proceeding, necessary concepts are introduced as follows:

Definition 1 (pattern space, see [25], page 44). Let $U$ be the universe of discourse. Suppose $\Phi$ is the discrete topology on $U$ (that is, the power set or the class of all subsets of $U$ ). Then, the pair $(U, \Phi)$ is referred to a pattern space.

Definition 2 (possibility space, see [25], page 44). Let $(U, \Phi)$ be a pattern space. A possibility measure $P_{\text {oss }}$ is a mapping from $\Phi$ to $[0,1]$ with the following properties:

(1) $P_{\text {oss }}(\varnothing)=0$.

(2) $P_{\text {oss }}(U)=1$.

(3) For an arbitrary collection of sets $A_{\alpha}$ of $\Phi$ (finite, countable, or uncountable), there holds

$$
P_{\text {oss }}\left(\cup_{\alpha} A_{\alpha}\right)=\sup _{\alpha} P_{\text {oss }}\left(A_{\alpha}\right) \text {. }
$$

Then, the triplet $\left(U, \Phi, P_{\text {oss }}\right)$ is referred to as a possibility space.

$$
\pi(A) \triangleq \sup _{u \in A} \pi_{X}(u)
$$

and $\pi_{X}(u)$ is the possibility distribution function of $\Pi_{X}$. This number, then, may be interpreted as the possibility that a value of $X$ belongs to $A$, that is,

$$
P_{o s s}\{X \in A\} \triangleq \pi(A) \triangleq \sup _{u \in A} \pi_{X}(u) .
$$

Definition 3 (possibility measure for classical set, see [9]). For a universe of discourse $U$ and $A$ is a classical set of $U$, let $\Pi_{X}$ be a possibility distribution associated with a variable $X$ which takes values in $U$. Then, the possibility measure, $\pi(A)$, is defined as a number in $[0,1]$ given by

Remark 1. The classical set is a set distinguishing from the fuzzy set. In classical set theory, the relationship between an element and a set is certain. However, in fuzzy set theory, the relationship between an element and a set is described by a membership function.

Definition 4 (unrelatedness, see [25], page 49). Suppose $\left(U, \Phi, P_{\text {oss }}\right)$ is a possibility space. $A_{1}, \ldots, A_{n} \in \Phi$ are said to be mutually unrelated if for any permutation of the set $\{1, \ldots, n\}$, denoted by $i_{1}, \ldots, i_{n}$, for $1 \leq k \leq n$, there holds

$$
P_{\text {oss }}\left(A_{i_{1}} \cap \ldots \cap A_{i_{k}}\right)=\min \left(P_{\text {oss }}\left(A_{i_{1}}\right), \ldots, P_{\text {oss }}\left(A_{i_{k}}\right)\right) \text {. }
$$

Definition 5. (system (component) lifetime, see [25], page 194). Let fuzzy variable $X$ be a real-valued function defined on a possibility space, i.e., $X: U \longrightarrow R^{+}=(0,+\infty)$, with the membership function $\mu_{X}: R^{+} \longrightarrow[0,1]$, then the lifetime of a system (component) is a nonnegative real-valued fuzzy variable, i.e., $X: U \longrightarrow R^{+}=(0,+\infty)$, with possibility distribution function

$$
\pi_{X}(x)=\mu_{X}(x)=P_{\text {oss }}(X=x), x \in R .
$$

Furthermore, $X$ is said to be normal/convex/strictly convex if the fuzzy set $\left\{x, \mu_{X}(x) ; x \in R^{+}\right\}$is normal/convex/ strictly convex.

Definition 6. (Posbist reliability, see [25], page 194). The Posbist reliability of a system is defined as the possibility that the system performs its assigned functions during a predefined exposure period under a given condition, which is denoted as

$$
\begin{aligned}
R_{e}(t) & =P_{o s s}(X>t)=\sup _{u>t} P_{o s s}(X=u) \\
& =\sup _{u>t} \mu_{X}(u), t \in R^{+} .
\end{aligned}
$$

Definition 7 (L-R fuzzy numbers, see [23]). Assume that $\widetilde{R}$ is a positive bounded and closed-interval of fuzzy numbers, whose membership function satisfies, $\forall \alpha>0$ and $\beta>0$ : 


$$
\mu_{R}(x)= \begin{cases}L\left(\frac{m_{-}-x}{\alpha}\right), & m_{-}-\alpha \leq x \leq m_{-}, \\ 1, & m_{-} \leq x \leq m_{+}, \\ R\left(\frac{x-m_{+}}{\beta}\right), & m_{+} \leq x \leq m_{+}+\beta, \\ 0, & \text { others, }\end{cases}
$$

where $m_{+}$and $m_{-}$are upper and lower modal values, respectively. $\left[m_{-}, m_{+}\right]$is peak range. $\alpha$ and $\beta$ are left and right scatters, respectively.

When $m_{-}=m_{+}=m_{0}$, the unique maximum value of the membership function will be achieved at the point $m_{0}$. The membership function is increasing on interval $\left(-\infty, m_{0}\right)$ and decreasing on interval $\left(m_{0},+\infty\right)$.

In practical application, $L(x)$ and $R(x)$ can be chosen as linear or nonlinear function, such as, triangular shape, normal shape, and sharp shape.

\section{Posbist Reliability Models of Typical Systems}

3.1. Posbist Reliability of Series System. Given a possibility space $\left(U, \Phi, P_{\text {oss }}\right)$, we consider a series system consisting of $n$ components, as shown in Figure 1. Suppose $X_{1}, X_{2}, \ldots, X_{n}$ are the lifetimes of the $n$ components, respectively. Then, the lifetime of system $X$ is $X=\min \left(X_{1}, X_{2}, \ldots, X_{n}\right)$.

Theorem 1. Given a possibility space $\left(U, \Phi, P_{\text {oss }}\right)$ and a series system consisting of $n$ components, the lifetime of components $X_{i}(i=1,2, \ldots, n)$ are unrelated and each a normal, strictly convex fuzzy variable, with continuous possibility distribution functions $\mu_{X_{1}}(x), \mu_{X_{2}}(x), \ldots, \mu_{X}(x)$, respectively. Let $X$ be the system lifetime, with possibility distribution function $\mu_{X}(x)$. Then, there exist $n$ positive numbers $a_{1}, a_{2}, \ldots, a_{n}$ (without loss of generality, we assume $\left.a_{1} \leq a_{2} \leq \cdots \leq a_{n}\right)$, such that the possibility distribution function of $X$ is

$$
\mu_{X}(x)= \begin{cases}\max \left\{\mu_{X_{1}}(x), \mu_{X_{2}}(x), \ldots, \mu_{X_{n}}(x)\right\}, & x \leq a_{1}, \\ \mu_{X_{1}}(x), & a_{1}<x \leq a_{2}, \\ \min \left\{\mu_{X_{1}}(x), \mu_{X_{2}}(x)\right\}, & a_{2}<x \leq a_{3}, \\ \ldots & \\ \min \left\{\mu_{X_{1}}(x), \mu_{X_{2}}(x), \ldots, \mu_{X_{n-1}}(x)\right\}, & a_{n-1}<x \leq a_{n}, \\ \min \left\{\mu_{X_{1}}(x), \mu_{X_{2}}(x), \ldots, \mu_{X_{n}}(x)\right\}, & x>a_{n} .\end{cases}
$$

Moreover, the Posbist reliability of the series system is

$$
R_{s}(t)=\sup _{u>t} \mu_{X}(u)= \begin{cases}1, & t \leq a_{1}, \\ \mu_{X_{1}}(t), & a_{1}<t \leq a_{2}, \\ \min \left\{\mu_{X_{1}}(t), \mu_{X_{2}}(t)\right\}, & a_{2}<t \leq a_{3}, \\ \ldots & \\ \min \left\{\mu_{X_{1}}(t), \mu_{X_{2}}(t), \ldots, \mu_{X_{n-1}}(t)\right\}, & a_{n-1}<t \leq a_{n}, \\ \min \left\{\mu_{X_{1}}(t), \mu_{X_{2}}(t), \ldots, \mu_{X_{n}}(t)\right\}, & t>a_{n} .\end{cases}
$$

Proof. Since the considered system is a series system and the lifetime of each component is $X_{i}(i=1,2, \ldots, n)$, then

$$
X=\min \left(X_{1}, X_{2}, \ldots, X_{n}\right) \text {. }
$$

For each component $X_{i}$, there exists a unique real

$$
\sup _{u \geq x} \mu_{X_{i}}(u)=\mid \begin{array}{ll}
1, & \text { if } x \leq a_{i} \\
\mu_{X_{i}}(x), & \text { if } x>a_{i}
\end{array}
$$

Without loss of generality, we assume $a_{1} \leq a_{2} \leq \cdots \leq a_{n}$. According to the definition of system lifetime,

$$
\begin{aligned}
\mu_{X}(x)= & P_{\text {oss }}\left(X=\min \left(X_{1}, X_{2}, \ldots, X_{n}\right)=x\right) \\
= & P_{\text {oss }}\left(X_{1}=x, X_{2} \geq x, X_{3} \geq x, \ldots, X_{n} \geq x\right) \\
& \cup P_{\text {oss }}\left(X_{1} \geq x, X_{2}=x, X_{3} \geq x, \ldots, X_{n} \geq x\right) \cup \cdots \cup P_{\text {oss }}\left(X_{1} \geq x, X_{2} \geq x, X_{3} \geq x, \ldots, X_{n}=x\right) \\
= & \max \left\{P_{\text {oss }}\left(X_{1}=x, X_{2} \geq x, X_{3} \geq x, \ldots, X_{n} \geq x\right),\right. \\
& \left.P_{\text {oss }}\left(X_{1} \geq x, X_{2}=x, X_{3} \geq x, \ldots, X_{n} \geq x\right), \ldots, P_{\text {oss }}\left(X_{1} \geq x, X_{2} \geq x, X_{3} \geq x, \ldots, X_{n}=x\right)\right\} .
\end{aligned}
$$




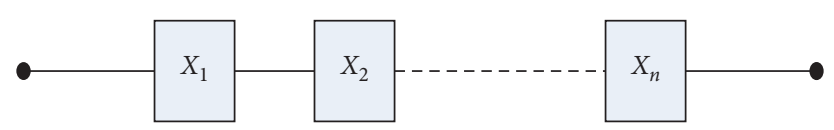

FIgURE 1: The logic block diagram of a series system.

In addition,

$$
\begin{aligned}
P_{o s s} & \left(X_{1} \geq x, \ldots, X_{i-1} \geq x, X_{i}=x, X_{i+1} \geq x, \ldots, X_{n} \geq x\right) \\
& =P_{o s s}\left(\cup_{a \geq x}\left\{X_{1}=a\right\}, \ldots, \cup_{a \geq x}\left\{X_{i-1}=a\right\}, X_{i}=x, \cup_{a \geq x}\left\{X_{i+1}=a\right\}, \ldots, \cup_{a \geq x}\left\{X_{n}=a\right\}\right) \\
& =P_{o s s}\left(\cup_{a \geq x}\left\{X_{1}=a, \ldots, X_{i-1}=a, X_{i}=x, X_{i+1}=a, \ldots, X_{n}=a\right\}\right) \\
& =\sup _{a \geq x}\left(P_{o s s} X_{1}=a, \ldots, X_{i-1}=a, X_{i}=x, X_{i+1}=a, \ldots, X_{n}=a\right) .
\end{aligned}
$$

Due to the unrelatedness of $X_{i}, i \in\{1,2, \ldots, n\}$, we have

$$
\begin{aligned}
& P_{\text {oss }}\left(X_{1} \geq x, \ldots, X_{i-1} \geq x, X_{i}=x, X_{i+1} \geq x, \ldots, X_{n} \geq x\right) \\
& =\sup _{a \geq x} \min \left(P_{\text {oss }}\left(X_{1}=a\right), \ldots, P_{\text {oss }}\left(X_{i-1}=a\right), P_{\text {oss }}\left(X_{i}=x\right), P_{\text {oss }}\left(X_{i+1}=a\right), \ldots, P_{\text {oss }}\left(X_{n}=a\right)\right) \\
& =\sup _{a \geq x} \min \left(\mu_{X_{1}}(a), \ldots, \mu_{X_{i-1}}(a), \mu_{X_{i}}(x), \mu_{X_{i+1}}(a), \ldots, \mu_{X_{n}}(a)\right) \\
& =\min \left(\sup _{a \geq x} \mu_{X_{1}}(a), \ldots, \sup _{a \geq x} \mu_{X_{i-1}}(a), \mu_{X_{i}}(x), \sup _{a \geq x} \mu_{X_{i+1}}(a), \ldots, \sup _{a \geq x} \mu_{X_{n}}(a)\right) \text {, } \\
& \left\{\min \left\{1, \ldots, 1, \mu_{X_{i}}(x), 1, \ldots, 1\right\}=\mu_{X_{i}}(x), \quad x \leq a_{1},\right. \\
& \min \left\{\mu_{X_{1}}(x), 1, \ldots, 1, \mu_{X_{i}}(x), 1, \ldots, 1\right\}=\min \left\{\mu_{X_{1}}(x), \mu_{X_{i}}(x)\right\}, \quad a_{1}<x \leq a_{2}, \\
& \min \left\{\mu_{X_{1}}(x), \mu_{X_{2}}(x), \ldots, \mu_{X_{i}}(x), 1, \ldots, 1\right\}=\min \left\{\mu_{X_{1}}(x), \mu_{X_{2}}(x), \ldots, \mu_{X_{i}}(x)\right\}, \quad a_{i-1}<x \leq a_{i}, \\
& = \begin{cases}\min \left\{\mu_{X_{1}}(x), \mu_{X_{2}}(x), \ldots, \mu_{X_{i}}(x), 1, \ldots, 1\right\}=\min \left\{\mu_{X_{1}}(x), \mu_{X_{2}}(x), \ldots, \mu_{X_{i}}(x)\right\}, \quad a_{i}<x \leq a_{i+1}, \\
\min \left\{\mu_{X_{1}}(x), \mu_{X_{2}}(x), \ldots, \mu_{X_{i+1}}(x), 1, \ldots, 1\right\}=\min \left\{\mu_{X_{1}}(x), \mu_{X_{2}}(x), \ldots, \mu_{X_{i+1}}(x)\right\}, a_{i+1}<x \leq a_{i+2}\end{cases} \\
& \min \left\{\mu_{X_{1}}(x), \mu_{X_{2}}(x), \ldots, \mu_{X_{i+1}}(x), 1, \ldots, 1\right\}=\min \left\{\mu_{X_{1}}(x), \mu_{X_{2}}(x), \ldots, \mu_{X_{i+1}}(x)\right\}, \quad a_{i+1}<x \leq a_{i+2}, \\
& \min \left\{\mu_{X_{1}}(x), \mu_{X_{2}}(x), \ldots, \mu_{X_{i+2}}(x), 1, \ldots, 1\right\}=\min \left\{\mu_{X_{1}}(x), \mu_{X_{2}}(x), \ldots, \mu_{X_{i+2}}(x)\right\}, \quad a_{i+2}<x \leq a_{i+3}, \\
& \text {-.. } \\
& \min \left\{\mu_{X_{1}}(x), \mu_{X_{2}}(x), \ldots, \mu_{X_{n-1}}(x), 1\right\}=\min \left\{\mu_{X_{1}}(x), \mu_{X_{2}}(x), \ldots, \mu_{X_{n-1}}(x)\right\}, \quad a_{n-1}<x \leq a_{n}, \\
& \min \left\{\mu_{X_{1}}(x), \mu_{X_{2}}(x), \ldots, \mu_{X_{n}}(x)\right\}, \quad x>a_{n} .
\end{aligned}
$$

Therefore,

$$
\begin{aligned}
\mu_{X}(x)= & \max \left\{\min \left(\mu_{X_{1}}(x), \sup _{a \geq x} \mu_{X_{2}}(a), \ldots, \sup _{a \geq x} \mu_{X_{n}}(a)\right)\right. \\
& \ldots, \min \left(\sup _{a \geq x} \mu_{X_{1}}(a), \ldots, \sup _{a \geq x} \mu_{X_{i-1}}(a), \mu_{X_{i}}(x),\right. \\
& \left.\sup _{a \geq x} \mu_{X_{i+1}}(a), \ldots, \sup _{a \geq x} \mu_{X_{n}}(a)\right), \ldots, \\
& \left.\min \left(\sup _{a \geq x} \mu_{X_{1}}(a), \ldots, \sup _{a \geq x} \mu_{X_{n-1}}(a), \mu_{X_{n}}(x)\right)\right\} .
\end{aligned}
$$

When $x \leq a_{1}$,

$$
\mu_{X}(x)=\max \left\{\mu_{X_{1}}(x), \mu_{X_{2}}(x), \ldots, \mu_{X_{n}}(x)\right\} .
$$

When $a_{1}<x \leq a_{2}$,

$$
\begin{aligned}
\mu_{X}(x)= & \max \left\{\mu_{X_{1}}(x), \min \left\{\mu_{X_{1}}(x), \mu_{X_{2}}(x)\right\} \min \left\{\mu_{X_{1}}(x), \mu_{X_{3}}(x)\right\}\right. \\
& \left.\ldots, \min \left\{\mu_{X_{1}}(x), \mu_{X_{n}}(x)\right\}\right\}, \\
= & \mu_{X_{1}}(x) .
\end{aligned}
$$

When $a_{2}<x \leq a_{3}$,

$$
\begin{aligned}
\mu_{X}(x)= & \max \left\{\min \left\{\mu_{X_{1}}(x), \mu_{X_{2}}(x)\right\},\right. \\
& \min \left\{\mu_{X_{1}}(x), \mu_{X_{2}}(x), \mu_{X_{3}}(x)\right\}, \\
& \min \left\{\mu_{X_{1}}(x), \mu_{X_{2}}(x), \mu_{X_{4}}(x)\right\}, \ldots, \\
& \left.\min \left\{\mu_{X_{1}}(x), \mu_{X_{2}}(x), \mu_{X_{n}}(x)\right\}\right\} \\
= & \min \left\{\mu_{X_{1}}(x), \mu_{X_{2}}(x)\right\}
\end{aligned}
$$


When $a_{3}<x \leq a_{4}$,

$$
\begin{aligned}
\mu_{X}(x)= & \max \left\{\min \left\{\mu_{X_{1}}(x), \mu_{X_{2}}(x), \mu_{X_{3}}(x)\right\}, \min \right. \\
& \left\{\mu_{X_{1}}(x), \mu_{X_{2}}(x), \mu_{X_{3}}(x), \mu_{X_{4}}(x)\right\}, \min \\
& \left\{\mu_{X_{1}}(x), \mu_{X_{2}}(x), \mu_{X_{3}}(x), \mu_{X_{5}}(x)\right\}, \ldots, \min \\
& \left.\left\{\mu_{X_{1}}(x), \mu_{X_{2}}(x), \mu_{X_{3}}(x), \mu_{X_{n}}(x)\right\}\right\} \\
= & \min \left\{\mu_{X_{1}}(x), \mu_{X_{2}}(x), \mu_{X_{3}}(x)\right\} .
\end{aligned}
$$

When $a_{i-1}<x \leq a_{i}$,

$$
\begin{aligned}
\mu_{X}(x)= & \max \left\{\min \left\{\mu_{X_{1}}(x), \ldots, \mu_{X_{i-1}}(x)\right\}, \min \right. \\
& \left\{\mu_{X_{1}}(x), \ldots, \mu_{X_{i-1}}(x), \mu_{X_{i}}(x)\right\}, \min \\
& \left\{\mu_{X_{1}}(x), \ldots, \mu_{X_{i-1}}(x), \mu_{X_{i+1}}(x)\right\}, \ldots, \min \\
& \left.\left\{\mu_{X_{1}}(x), \ldots, \mu_{X_{i-1}}(x), \mu_{X_{n}}(x)\right\}\right\} \\
= & \min \left\{\mu_{X_{1}}(x), \ldots, \mu_{X_{i-1}}(x)\right\} .
\end{aligned}
$$

When $a_{n-1}<x \leq a_{n}$,

$$
\begin{aligned}
\mu_{X}(x)= & \max \left\{\min \left\{\mu_{X_{1}}(x), \mu_{X_{2}}(x), \ldots, \mu_{X_{n-1}}(x)\right\}, \min \right. \\
& \left.\left\{\mu_{X_{1}}(x), \mu_{X_{2}}(x), \ldots, \mu_{X_{n-1}}(x), \mu_{X_{n}}(x)\right\}\right\} \\
= & \min \left\{\mu_{X_{1}}(x), \ldots, \mu_{X_{n-1}}(x)\right\} .
\end{aligned}
$$

When $x>a_{n}$,

$$
\mu_{X}(x)=\min \left\{\mu_{X_{1}}(x), \mu_{X_{2}}(x), \ldots, \mu_{X_{n}}(x)\right\} .
$$

So, we have

$$
\mu_{X}(x)= \begin{cases}\max \left\{\mu_{X_{1}}(x), \mu_{X_{2}}(x), \ldots, \mu_{X_{n}}(x)\right\}, & x \leq a_{1}, \\ \mu_{X_{1}}(x), & a_{1}<x \leq a_{2}, \\ \min \left\{\mu_{X_{1}}(x), \mu_{X_{2}}(x)\right\}, & a_{2}<x \leq a_{3}, \\ \ldots & \\ \min \left\{\mu_{X_{1}}(x), \mu_{X_{2}}(x), \ldots, \mu_{X_{n-1}}(x)\right\}, & a_{n-1}<x \leq a_{n}, \\ \min \left\{\mu_{X_{1}}(x), \mu_{X_{2}}(x), \ldots, \mu_{X_{n}}(x)\right\}, & x>a_{n} .\end{cases}
$$

Since the lifetime of components $X_{i}(i=1,2, \ldots, n)$ are unrelated and each a normal, strictly convex fuzzy variable, with continuous possibility distribution functions $\mu_{X_{1}}(x), \mu_{X_{2}}(x), \ldots, \mu_{X_{n}}(x)$, respectively, $a_{i}$ is a unique real number such that $\sup _{u \in R^{+}} \mu_{X_{i}}(u)=\mu_{X_{i}}\left(a_{i}\right)=1$. When $u \leq a_{i}$, $\mu_{X_{i}}(u)$ is a strictly monotonically increasing function. When $u>a_{i}, \mu_{X_{i}}(u)$ is a strictly monotonically decreasing function. When $a_{i}=+\infty, \mu_{X_{i}}(u)$ is a strictly monotonically increasing function in the whole domain.

The Posbist reliability of series system can be expressed as $R_{s}(t)=\sup _{u>t} \mu_{X}(u)$; combining with (11), we have when $t \leq a_{1}$,

$$
\begin{aligned}
R_{s}(t)= & \sup _{u>t} \mu_{X}(u) \\
= & \max \left\{\sup _{y \in\left(t, a_{1}\right]} \mu_{X}(y), \sup _{y \in\left(a_{1}, a_{2}\right]} \mu_{X}(y), \ldots, \sup _{y \in\left(a_{n},+\infty\right)} \mu_{X}(y)\right\} \\
= & \max \left\{\sup _{y \in\left(t, a_{1}\right]} \max \left\{\mu_{X_{1}}(y), \mu_{X_{2}}(y), \ldots, \mu_{X_{n}}(y)\right\}, \sup _{y \in\left(a_{1}, a_{2}\right]} \mu_{X_{1}}(y), \ldots, \sup _{y \in\left(a_{n},+\infty\right)} \min \right. \\
& \left.\left\{\mu_{X_{1}}(y), \mu_{X_{2}}(y), \ldots, \mu_{X_{n}}(y)\right\}\right\},
\end{aligned}
$$

where

$$
\begin{aligned}
& \sup _{y \in\left(t, a_{1}\right]} \max \left\{\mu_{X_{1}}(y), \mu_{X_{2}}(y), \ldots, \mu_{X_{n}}(y)\right\} \\
& =\max \left\{\sup _{y \in\left(t, a_{1}\right]} \mu_{X_{1}}(y), \sup _{y \in\left(t, a_{1}\right]} \mu_{X_{2}}\right. \\
& \left.(y), \ldots, \sup _{y \in\left(t, a_{1}\right]} \mu_{X_{n}}(y)\right\} .
\end{aligned}
$$

Since $\sup _{y>t} \mu_{X_{1}}(y)=1\left(t \leq a_{1}\right)$, then

$$
\sup _{y>t} \mu_{X_{1}}(y)=\sup _{y \in\left(t, a_{1}\right] \cup y \in\left(a_{1},+\infty\right)} \mu_{X_{1}}(y)
$$

$$
\begin{aligned}
& =\max \left\{\sup _{y \in\left(t, a_{1}\right]} \mu_{X_{1}}(y), \sup _{y \in\left(a_{1},+\infty\right)} \mu_{X_{1}}(y)\right\} \\
& =\max \left\{\sup _{y \in\left(t, a_{1}\right]} \mu_{X_{1}}(y), \mu_{X_{1}}\left(a_{1}^{+}\right)\right\}=1 .
\end{aligned}
$$

So,

$$
\sup _{y \in\left(t, a_{1}\right]} \mu_{X_{1}}(y)=1
$$


No matter what the remaining terms' value is, the maximum value of all terms is 1 .

Therefore,

$$
R_{s}(t)=1 \text {. }
$$

When $a_{1}<t \leq a_{2}$,

$$
\begin{aligned}
R_{s}(t)= & \sup _{u>t} \mu_{X}(u)=\max \left\{\sup _{y \in\left(t, a_{2}\right]} \mu_{X}(y), \sup _{y \in\left(a_{2}, a_{3}\right]} \mu_{X}(y), \ldots, \sup _{y \in\left(a_{n},+\infty\right)} \mu_{X}(y)\right\} \\
= & \max \left\{\sup _{y \in\left(t, a_{2}\right]} \mu_{X_{1}}(y), \sup _{y \in\left(a_{2}, a_{3}\right]} \min \left\{\mu_{X_{1}}(y), \mu_{X_{2}}(y)\right\}, \ldots, \sup _{y \in\left(a_{n},+\infty\right)} \min \left\{\left\{\mu_{X_{1}}(y), \mu_{X_{2}}(y), \ldots, \mu_{X_{n}}(y)\right\}\right\}\right. \\
= & \max \left\{\sup _{y \in\left(t, a_{2}\right]} \mu_{X_{1}}(y), \min \left\{\sup _{y \in\left(a_{2}, a_{3}\right]} \mu_{X_{1}}(y), \sup _{y \in\left(a_{2}, a_{3}\right]} \mu_{X_{2}}(y)\right\}, \ldots, \min \right. \\
& \left.\cdot\left\{\sup _{y \in\left(a_{n},+\infty\right)} \mu_{X_{1}}(y), \sup _{y \in\left(a_{n},+\infty\right)} \mu_{X_{2}}(y), \ldots, \sup _{y \in\left(a_{n},+\infty\right)} \mu_{X_{n}}(y)\right\}\right\},
\end{aligned}
$$

where

$$
\sup _{y \in\left(t, a_{2}\right]} \mu_{X_{1}}(y)=\mu_{X_{1}}(t) \text {. }
$$

If

$$
\sup _{y \in\left(a_{2}, a_{3}\right]} \mu_{X_{1}}(y) \leq \sup _{y \in\left(a_{2}, a_{3}\right]} \mu_{X_{2}}(y),
$$

then

$$
\begin{aligned}
& \min \left\{\sup _{y \in\left(a_{2}, a_{3}\right]} \mu_{X_{1}}(y), \sup _{y \in\left(a_{2}, a_{3}\right]} \mu_{X_{2}}(y)\right\} \\
& =\sup _{y \in\left(a_{2}, a_{3}\right]} \mu_{X_{1}}(y) \leq \mu_{X_{1}}(y) .
\end{aligned}
$$

Otherwise,

$$
\begin{aligned}
& \min \left\{\sup _{y \in\left(a_{2}, a_{3}\right]} \mu_{X_{1}}(y), \sup _{y \in\left(a_{2}, a_{3}\right]} \mu_{X_{2}}(y)\right\} \\
& \quad=\sup _{y \in\left(a_{2}, a_{3}\right]} \mu_{X_{2}}(y) \leq \sup _{y \in\left(a_{2}, a_{3}\right]} \mu_{X_{1}}(y) \\
& \quad \leq \sup _{y \in\left(t, a_{2}\right]} \mu_{X_{1}}(y) \leq \mu_{X_{1}}(y) .
\end{aligned}
$$

Similarly, for $i \in\{3,4, \ldots, n\}$, when $i=n$, let $i+1=+\infty$ and the interval becomes a open set:

$$
\begin{aligned}
& \min \left\{\sup _{y \in\left(a_{i}, a_{i+1}\right]} \mu_{X_{1}}(y), \sup _{y \in\left(a_{i}, a_{i+1}\right]} \mu_{X_{2}}\right. \\
& \left.\cdot(y), \ldots, \sup _{y \in\left(a_{i}, a_{i+1}\right]} \mu_{X_{i}}(y)\right\} \\
& \quad \leq \mu_{X_{i}}(t) .
\end{aligned}
$$

Therefore,

$$
R_{s}(t)=\mu_{X_{1}}(t) .
$$

When $a_{n-1}<t \leq a_{n}$,

$$
\begin{aligned}
& R_{s}(t)=\sup _{u>t} \mu_{X}(u)=\max \left\{\sup _{y \in\left(t, a_{n}\right]} \mu_{X}(y), \sup _{y \in\left(a_{n},+\infty\right)} \mu_{X}(y)\right\} \\
& =\max \left\{\sup _{y \in\left(t, a_{n}\right]} \min \left\{\left\{\mu_{X_{1}}(y), \mu_{X_{2}}(y), \ldots, \mu_{X_{n-1}}(y)\right\}, \sup _{y \in\left(a_{n},+\infty\right)} \min \left\{\mu_{X_{1}}(y), \mu_{X_{2}}(y), \ldots, \mu_{X_{n}}(y)\right\}\right\}\right. \\
& =\max \left\{\min \left\{\sup _{y \in\left(t, a_{n}\right]} \mu_{X_{1}}(y), \sup _{y \in\left(t, a_{n}\right]} \mu_{X_{2}}(y), \ldots, \sup _{y \in\left(t, a_{n}\right]} \mu_{X_{n-1}}(y)\right\}, \min \right. \\
& \left.\cdot\left\{\sup _{y \in\left(a_{n},+\infty\right)} \mu_{X_{1}}(y), \sup _{y \in\left(a_{n},+\infty\right)} \mu_{X_{2}}(y), \ldots, \sup _{y \in\left(a_{n},+\infty\right)} \mu_{X_{n}}(y)\right\}\right\} \\
& =\min \left\{\mu_{X_{1}}(t), \mu_{X_{2}}(t), \ldots, \mu_{X_{n-1}}(t)\right\} .
\end{aligned}
$$


When $t>a_{n}$,

$$
\begin{aligned}
R_{s}(t)= & \sup _{u>t} \mu_{X}(u)=\sup _{u>t} \min \left\{\left\{\mu_{X_{1}}(u), \mu_{X_{2}}(u), \ldots, \mu_{X_{n}}(u)\right\}\right. \\
& =\min \left\{\sup _{u>t} \mu_{X_{1}}(u), \sup _{u>t} \mu_{X_{2}}(u), \ldots, \sup _{u>t} \mu_{X_{n}}(u)\right\} \\
& =\min \left\{\mu_{X_{1}}(t), \mu_{X_{2}}(t), \ldots, \mu_{X_{n}}(t)\right\} .
\end{aligned}
$$

Therefore, the Posbist reliability of the series system is

$$
R_{s}(t)=\sup _{u>t} \mu_{X}(u)= \begin{cases}1, & t \leq a_{1}, \\ \mu_{X_{1}}(t), & a_{1}<t \leq a_{2}, \\ \min \left\{\mu_{X_{1}}(t), \mu_{X_{2}}(t)\right\}, & a_{2}<t \leq a_{3}, \\ \ldots & \\ \min \left\{\mu_{X_{1}}(t), \mu_{X_{2}}(t), \ldots, \mu_{X_{n-1}}(t)\right\}, & a_{n-1}<t \leq a_{n}, \\ \min \left\{\mu_{X_{1}}(t), \mu_{X_{2}}(t), \ldots, \mu_{X_{n}}(t)\right\}, & t>a_{n} .\end{cases}
$$

Specially, when the lifetime of the components are assumed as symmetrical Gaussian fuzzy variables, i.e., $L(x)$ and $R(x)$ have a normal distribution. We have the following corollary.

Corollary 1. Given a possibility space $\left(U, \Phi, P_{\text {oss }}\right)$ and a series system consisting of $n$ components, the possibility distribution function of the lifetime of components $X_{i}(i=1,2, \ldots, n)$ are assumed to be

$$
\mu_{X_{i}}(x)=\exp \left(-\left(\frac{x-m_{i}}{h}\right)^{2}\right)
$$

without loss of generality; we assume $m_{1} \leq m_{2} \leq \cdots \leq m_{n}$; then, the possibility distribution function of the system lifetime $X$ is

$$
\mu_{X}(x)=\exp \left(-\left(\frac{x-m_{1}}{h}\right)^{2}\right)
$$

Moreover, the Posbist reliability of the series system is

$$
R_{s}(t)=\sup _{u>t} \mu_{X}(u)= \begin{cases}1, & t \leq m_{1}, \\ \exp \left(-\left(\frac{t-m_{1}}{h}\right)^{2}\right), & t>m_{1} .\end{cases}
$$

Remark 2. Given a possibility space $\left(U, \Phi, P_{\text {oss }}\right)$ and a series system consisting of $n$ components, assume the possibility distribution function of the lifetime of components $X_{i}(i=$ $1,2, \ldots, n)$ are identically and independently distributed ones $\mu_{X_{1}}(x)$; then, there exists a real number $a$, such that $\mu_{X}(x)=\mu_{X_{1}}(x)$
$R_{s}(t)=\sup _{u>t} \mu_{X}(u)=\left\{\begin{array}{ll}1 & t \leq a \\ \mu_{X_{1}}(t) & t>a\end{array}\right.$.

and space $\left(U, \Phi, P_{\text {oss }}\right)$, we consider a parallel system consisting of $n$ components, as shown in Figure 2 . Suppose $X$ is the system lifetime and $X_{1}, X_{2}, \ldots, X_{n}$ are the lifetimes of $n$ components, respectively. Then, the lifetime of system $X$ is $X=\operatorname{ma}\left(X_{1}, X_{2}, \ldots, X_{n}\right)$.

Theorem 2. Given a possibility space $\left(U, \Phi, P_{\text {oss }}\right)$ and a parallel system consisting of $n$ components, the lifetime of components $X_{i}(i=1,2, \ldots, n)$ are unrelated and each a normal, strictly convex fuzzy variable, with continuous possibility distribution functions $\mu_{X_{1}}(x), \mu_{X_{2}}(x), \ldots, \mu_{X_{n}}(x)$, respectively. Let the system lifetime be $X$ with possibility distribution function $\mu_{X}(x)$. Then, there exist $n$ positive numbers $a_{1}, a_{2}, \ldots, a_{n}$ (without loss of generality, we assume $a_{1} \leq a_{2} \leq \cdots \leq a_{n}$ ), such that the possibility distribution function of $X$ is

$$
\mu_{X}(x)= \begin{cases}\min \left\{\mu_{X_{1}}(x), \mu_{X_{2}}(x), \ldots, \mu_{X_{n}}(x)\right\}, & x \leq a_{1}, \\ \min \left\{\mu_{X_{2}}(x), \mu_{X_{3}}(x), \ldots, \mu_{X_{n}}(x)\right\}, & a_{1}<x \leq a_{2}, \\ \min \left\{\mu_{X_{3}}(x), \mu_{X_{4}}(x), \ldots, \mu_{X_{n}}(x)\right\}, & a_{2}<x \leq a_{3}, \\ \ldots & \\ \mu_{X_{n}}(x), a_{n-1}<x \leq a_{n}, & \\ \max \left\{\mu_{X_{1}}(x), \mu_{X_{2}}(x), \ldots, \mu_{X_{n}}(x)\right\}, & x>a_{n} .\end{cases}
$$

Moreover, the Posbist reliability of the parallel system is $R_{p}(t)=\sup _{u>t} \mu_{X}(u)= \begin{cases}1, & t \leq a_{n}, \\ \max \left\{\mu_{X_{1}}(t), \mu_{X_{2}}(t), \ldots, \mu_{X_{n}}(t)\right\}, & t>a_{n} .\end{cases}$

Proof. Similar to Theorem 1, for each component $X_{i}$, there exists a unique real number $a_{i} \in R^{+}$, such that 


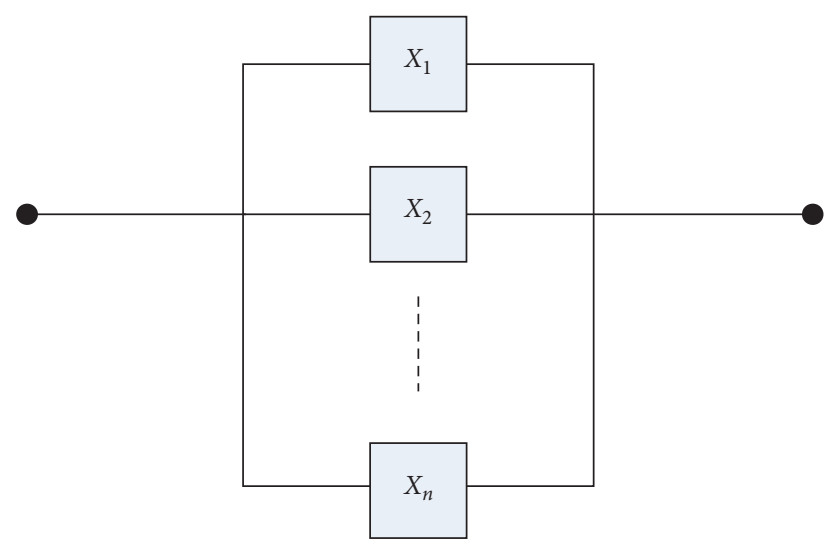

FIgURE 2: The logic block diagram of a parallel system.

$$
\sup _{u \leq x} \mu_{X_{i}}(u)= \begin{cases}\mu_{X_{i}}(x), & \text { if } x \leq a_{i} \\ 1, & \text { if } x>a_{i}\end{cases}
$$

Due to the assumption $a_{1} \leq a_{2} \leq \cdots \leq a_{n}$ and combining with the definition of system lifetime, we have

$$
\begin{aligned}
\mu_{X}(x)= & P_{\text {oss }}\left(X=\max \left(\left(X_{1}, X_{2}, \ldots, X_{n}\right)\right)=x\right) \\
= & \max \left\{P_{\text {oss }}\left(X_{1}=x, X_{2} \leq x, X_{3} \leq x, \ldots, X_{n} \leq x\right),\right. \\
& P_{\text {oss }}\left(X_{1} \leq x, X_{2}=x, X_{3} \leq x, \ldots, X_{n} \leq x\right), \ldots, P_{\text {oss }} \\
& \left.\cdot\left(X_{1} \leq x, X_{2} \leq x, X_{3} \leq x, \ldots, X_{n}=x\right)\right\},
\end{aligned}
$$

where

$$
\begin{aligned}
& P_{\text {oss }}\left(X_{1} \leq x, \ldots, X_{i-1} \leq x, X_{i}=x, X_{i+1} \leq x, \ldots, X_{n} \leq x\right) \\
& \quad=\sup _{a \leq x} P_{\text {oss }}\left(X_{1}=a, \ldots, X_{i-1}=a, X_{i}=x, X_{i+1}=a, \ldots, X_{n}=a\right) .
\end{aligned}
$$

Because of the unrelatedness of $X_{i}, i \in\{1,2, \ldots, n\}$, we have

$$
\begin{aligned}
& P_{o s s}\left(X_{1} \leq x, \ldots, X_{i-1} \leq x, X_{i}=x, X_{i+1} \leq x, \ldots, X_{n} \leq x\right) \\
&= \sup _{a \leq x} \min \left(P_{o s s}\left(X_{1}=a\right), \ldots, P_{o s s}\left(X_{i-1}=a\right), P_{o s s}\left(X_{i}=x\right), P_{o s s}\left(X_{i+1}=a\right), \ldots, P_{o s s}\left(X_{n}=a\right)\right) \\
&=\sup _{a \leq x} \min \left(\mu_{X_{1}}(a), \ldots, \mu_{X_{i-1}}(a), \mu_{X_{i}}(x), \mu_{X_{i+1}}(a), \ldots, \mu_{X_{n}}(a)\right) \\
& \min \left(\sup _{a \leq x} \mu_{X_{1}}(a), \ldots, \sup _{a \leq x} \mu_{X_{i-1}}(a), \mu_{X_{i}}(x), \sup _{a \leq x} \mu_{X_{i+1}}(a), \ldots, \sup _{a \leq x} \mu_{X_{n}}(a)\right) \\
& \min \left\{\mu_{X_{1}}(x), \mu_{X_{2}}(x), \ldots, \mu_{X_{i}}(x), \ldots, \mu_{X_{n}}(x)\right\}, x \leq a_{1} \\
& \min \left\{1, \mu_{X_{2}}(x), \mu_{X_{3}}(x), \ldots, \mu_{X_{i}}(x), \ldots, \mu_{X_{n}}(x)\right\}=\min \left\{\mu_{X_{2}}(x), \mu_{X_{3}}(x), \ldots, \mu_{X_{n}}(x)\right\}, a_{1}<x \leq a_{2}, \\
& \min \left\{1, \ldots, 1, \mu_{X_{i}}(x), \ldots, \mu_{X_{n}}(x)\right\}=\min \left\{\mu_{X_{i}}(x), \mu_{X_{i+1}}(x), \ldots, \mu_{X_{n}}(x)\right\}, a_{i-1}<x \leq a_{i}, \\
& \min \left\{1, \ldots, 1, \mu_{X_{i}}(x), \ldots, \mu_{X_{n}}(x)\right\}=\min \left\{\mu_{X_{i}}(x), \mu_{X_{i+1}}(x), \ldots, \mu_{X_{n}}(x)\right\}, a_{i}<x \leq a_{i+1}, \\
& \min \left\{1, \ldots, 1, \mu_{X_{i}}(x), 1, \mu_{X_{i+2}}(x), \ldots, \mu_{X_{n}}(x)\right\}=\min \left\{\mu_{X_{i}}(x), \mu_{X_{i+2}}(x), \ldots, \mu_{X_{n}}(x)\right\}, a_{i+1}<x \leq a_{i+2}, \\
& \min \left\{1, \ldots, 1, \mu_{X_{i}}(x), 1,1, \mu_{X_{i+3}}(x), \ldots, \mu_{X_{n}}(x)\right\}=\min \left\{\mu_{X_{i}}(x), \mu_{X_{i+3}}(x), \ldots, \mu_{X_{n}}(x)\right\}, a_{i+2}<x \leq a_{i+3}, \\
& \ldots \\
& \min \left\{1, \ldots, 1, \mu_{X_{i}}(x), 1, \ldots, 1, \mu_{X_{n}}(x)\right\}=\min \left\{\mu_{X_{i}}(x), \mu_{X_{n}}(x)\right\}, a_{n-1}<x \leq a_{n},
\end{aligned}
$$

Therefore,

$$
\begin{aligned}
\mu_{X}(x)= & \max \left\{\min \left(\mu_{X_{1}}(x), \sup _{a \leq x} \mu_{X_{2}}(a), \ldots, \sup _{a \leq x} \mu_{X_{n}}(a)\right), \ldots, \min \right. \\
& \cdot\left(\sup _{a \leq x} \mu_{X_{1}}(a), \ldots, \sup _{a \leq x} \mu_{X_{i-1}}(a), \mu_{X_{i}}(x), \sup _{a \leq x} \mu_{X_{i+1}}(a), \ldots, \sup _{a \leq x} \mu_{X_{n}}(a)\right), \ldots, \min \\
& \left.\cdot\left(\sup _{a \leq x} \mu_{X_{1}}(a), \ldots, \sup _{a \leq x} \mu_{X_{n-1}}(a), \mu_{X_{n}}(x)\right)\right\} .
\end{aligned}
$$


When $x \leq a_{1}$,

$$
\mu_{X}(x)=\min \left\{\mu_{X_{1}}(x), \mu_{X_{2}}(x), \ldots, \mu_{X_{n}}(x)\right\} .
$$

When $a_{1}<x \leq a_{2}$,

$$
\begin{aligned}
\mu_{X}(x)= & \max \left\{\min \left\{\mu_{X_{1}}(x), \mu_{X_{2}}(x), \ldots, \mu_{X_{n}}(x)\right\}, \min \right. \\
& \left.\cdot\left\{\mu_{X_{2}}(x), \ldots, \mu_{X_{n}}(x)\right\}, \ldots, \min \left\{\mu_{X_{2}}(x), \ldots, \mu_{X_{n}}(x)\right\}\right\} \\
= & \min \left\{\mu_{X_{2}}(x), \ldots, \mu_{X_{n}}(x)\right\} .
\end{aligned}
$$

When $a_{2}<x \leq a_{3}$,

$$
\begin{aligned}
\mu_{X}(x)= & \max \left\{\min \left\{\mu_{X_{1}}(x), \mu_{X_{3}}(x), \ldots, \mu_{X_{n}}(x)\right\}, \min \right. \\
& \cdot\left\{\mu_{X_{2}}(x), \ldots, \mu_{X_{n}}(x)\right\}, \min \\
& \cdot\left\{\mu_{X_{3}}(x), \ldots, \mu_{X_{n}}(x)\right\}, \ldots, \min \\
& \left.\cdot\left\{\mu_{X_{3}}(x), \ldots, \mu_{X_{n}}(x)\right\}\right\} \\
= & \min \left\{\mu_{X_{3}}(x), \ldots, \mu_{X_{n}}(x)\right\} .
\end{aligned}
$$

When $a_{3}<x \leq a_{4}$,

$$
\begin{aligned}
\mu_{X}(x)= & \max \left\{\min \left\{\mu_{X_{1}}(x), \mu_{X_{4}}(x), \ldots, \mu_{X_{n}}(x)\right\}, \min \right. \\
& \cdot\left\{\mu_{X_{2}}(x), \mu_{X_{4}}(x), \ldots, \mu_{X_{n}}(x)\right\}, \min \\
& \cdot\left\{\mu_{X_{3}}(x), \mu_{X_{4}}(x), \ldots, \mu_{X_{n}}(x)\right\}, \min \\
& \cdot\left\{\mu_{X_{4}}(x), \ldots, \mu_{X_{n}}(x)\right\}, \ldots, \min \\
& \left.\cdot\left\{\mu_{X_{4}}(x), \ldots, \mu_{X_{n}}(x)\right\}\right\} \\
= & \min \left\{\mu_{X_{4}}(x), \ldots, \mu_{X_{n}}(x)\right\} .
\end{aligned}
$$

When $a_{i-1}<x \leq a_{i}$,

$$
\begin{aligned}
\mu_{X}(x)= & \max \left\{\min \left\{\mu_{X_{1}}(x), \mu_{X_{i}}(x), \ldots, \mu_{X_{n}}(x)\right\}, \min \right. \\
& \cdot\left\{\mu_{X_{2}}(x), \mu_{X_{i}}(x), \ldots, \mu_{X_{n}}(x)\right\}, \ldots, \min \\
& \cdot\left\{\mu_{X_{i-1}}(x), \mu_{X_{i}}(x), \ldots, \mu_{X_{n}}(x)\right\}, \min \\
& \cdot\left\{\mu_{X_{i}}(x), \ldots, \mu_{X_{n}}(x)\right\}, \ldots, \min \\
& \left.\cdot\left\{\mu_{X_{i}}(x), \ldots, \mu_{X_{n}}(x)\right\}\right\} \\
= & \min \left\{\mu_{X_{i}}(x), \ldots, \mu_{X_{n}}(x)\right\} .
\end{aligned}
$$

When $a_{n-1}<x \leq a_{n}$,

$$
\begin{aligned}
\mu_{X}(x)= & \max \left\{\min \left\{\mu_{X_{1}}(x), \mu_{X_{n}}(x)\right\}, \min \right. \\
& \cdot\left\{\mu_{X_{2}}(x), \mu_{X_{n}}(x)\right\}, \ldots, \min \\
& \left.\cdot\left\{\mu_{X_{n-1}}(x), \mu_{X_{n}}(x)\right\}, \mu_{X_{n}}(x)\right\} \\
= & \mu_{X_{n}}(x) .
\end{aligned}
$$

When $x>a_{n}$,

$$
\mu_{X}(x)=\max \left\{\mu_{X_{1}}(x), \mu_{X_{2}}(x), \ldots, \mu_{X_{n}}(x)\right\} .
$$

So, we have

$$
\mu_{X}(x)= \begin{cases}\min \left\{\mu_{X_{1}}(x), \mu_{X_{2}}(x), \ldots, \mu_{X_{n}}(x)\right\}, & x \leq a_{1}, \\ \min \left\{\mu_{X_{2}}(x), \mu_{X_{3}}(x), \ldots, \mu_{X_{n}}(x)\right\}, & a_{1}<x \leq a_{2}, \\ \min \left\{\mu_{X_{3}}(x), \mu_{X_{4}}(x), \ldots, \mu_{X_{n}}(x)\right\}, & a_{2}<x \leq a_{3}, \\ \ldots & \\ \mu_{X_{n}}(x), a_{n-1}<x \leq a_{n} & \\ \max \left\{\mu_{X_{1}}(x), \mu_{X_{2}}(x), \ldots, \mu_{X_{n}}(x)\right\}, & x>a_{n} .\end{cases}
$$

The Posbist reliability of the parallel system is

$$
R_{p}(t)=\sup _{u>t} \mu_{X}(u) .
$$

Since the point $a_{i}$ is a unique maximum value point, based on the monotonicity of $\mu_{X_{i}}(x)$, one has when $t \leq a_{1}$ 


$$
\begin{aligned}
& R_{p}(t)=\sup _{u>t} \mu_{X}(u)=\sup _{\left(t, a_{1}\right] \cup\left(a_{1}, a_{2}\right] \cup \ldots \cup\left(a_{n},+\infty\right)} \mu_{X}(u) \\
& =\max \left\{\operatorname { s u p } _ { y \in ( t , a _ { 1 } ] } \operatorname { m i n } \left\{\left\{\mu_{X_{1}}(y), \mu_{X_{2}}(y), \ldots, \mu_{X_{n}}(y)\right\}, \sup _{y \in\left(a_{1}, a_{2}\right]} \min \right.\right. \\
& \left.\cdot\left\{\mu_{X_{2}}(y), \mu_{X_{3}}(y), \ldots, \mu_{X_{n}}(y)\right\}, \ldots, \sup _{y \in\left(a_{n-1}, a_{n}\right]} \mu_{X_{n}}(y), \sup _{y \in\left(a_{n},+\infty\right)} \max \left\{\mu_{X_{1}}(y), \mu_{X_{2}}(y), \ldots, \mu_{X_{n}}(y)\right\}\right\} \\
& =\max \left\{\min \left\{\sup _{y \in\left(t, a_{1}\right]} \mu_{X_{1}}(y), \ldots, \sup _{y \in\left(t, a_{2}\right]} \mu_{X_{n}}(y)\right\}, \min \right. \\
& \cdot\left\{\sup _{y \in\left(a_{1}, a_{2}\right]} \mu_{X_{2}}(y), \ldots, \sup _{y \in\left(a_{1}, a_{2}\right]} \mu_{X_{n}}(y)\right\}, \ldots, \sup _{y \in\left(a_{n-1}, a_{n}\right]} \mu_{X_{n}}(y), \max \\
& \left.\cdot\left\{\sup _{y \in\left(a_{n},+\infty\right)} \mu_{X_{1}}(y), \sup _{y \in\left(a_{n},+\infty\right)} \mu_{X_{2}}(y), \ldots, \sup _{y \in\left(a_{n},+\infty\right)} \mu_{X_{n}}(y)\right\}\right\} \\
& =\max \left\{\min \left\{\sup _{y \in\left(t, a_{1}\right]} \mu_{X_{1}}(y), \sup _{y \in\left(t, a_{2}\right]} \mu_{X_{2}}(y), \ldots, \sup _{y \in\left(t, a_{n}\right]} \mu_{X_{n}}(y)\right\}, \max \right. \\
& \left.\cdot\left\{\sup _{y \in\left(a_{n},+\infty\right)} \mu_{X_{1}}(y), \sup _{y \in\left(a_{n},+\infty\right)} \mu_{X_{2}}(y), \ldots, \sup _{y \in\left(a_{n},+\infty\right)} \mu_{X_{n}}(y)\right\}\right\} \\
& =1 \text {. }
\end{aligned}
$$

When $a_{1}<t \leq a_{2}$,

$$
\begin{aligned}
& R_{p}(t)=\sup _{u>t} \mu_{X}(u)=\sup _{\left(t, a_{2}\right] \cup\left(a_{2}, a_{3}\right] \cup \cdots \cup\left(a_{n},+\infty\right)} \mu_{X}(u) \\
& =\max \left\{\sup _{y \in\left(t, a_{2}\right]} \min \left\{\mu_{X_{2}}(y), \mu_{X_{3}}(y), \ldots, \mu_{X_{n}}(y)\right\}, \sup _{y \in\left(a_{2}, a_{3}\right]} \min \right. \\
& \left.\cdot\left\{\mu_{X_{3}}(y), \mu_{X_{4}}(y), \ldots, \mu_{X_{n}}(y)\right\}, \ldots, \sup _{y \in\left(a_{n-1}, a_{n}\right]} \mu_{X_{n}}(y), \sup _{y \in\left(a_{n},+\infty\right)} \max \left\{\mu_{X_{1}}(y), \mu_{X_{2}}(y), \ldots, \mu_{X_{n}}(y)\right\}\right\} \\
& =\max \left\{\operatorname { m i n } \left\{\left\{\sup _{y \in\left(t, a_{2}\right]} \mu_{X_{2}}(y), \ldots, \sup _{y \in\left(t, a_{2}\right]} \mu_{X_{n}}(y)\right\}, \min \right.\right. \\
& \cdot\left\{\sup _{y \in\left(a_{2}, a_{3}\right]} \mu_{X_{3}}(y), \ldots, \sup _{y \in\left(a_{2}, a_{3}\right]} \mu_{X_{n}}(y)\right\}, \ldots, \sup _{y \in\left(a_{n-1}, a_{n}\right]} \mu_{X_{n}}(y), \max \\
& \left.\cdot\left\{\sup _{y \in\left(a_{n},+\infty\right)} \mu_{X_{1}}(y), \sup _{y \in\left(a_{n},+\infty\right)} \mu_{X_{2}}(y), \ldots, \sup _{y \in\left(a_{n},+\infty\right)} \mu_{X_{n}}(y)\right\}\right\} \\
& =\max \left\{\min \left\{\sup _{y \in\left(t, a_{2}\right]} \mu_{X_{2}}(y), \operatorname{ssup}_{y \in\left(t, a_{3}\right]} \mu_{X_{3}}(y), \ldots, \sup _{y \in\left(t, a_{n}\right]} \mu_{X_{n}}(y)\right\}\right\}, \max \\
& \left.\cdot\left\{\sup _{y \in\left(a_{n},+\infty\right)} \mu_{X_{1}}(y), \sup _{y \in\left(a_{n},+\infty\right)} \mu_{X_{2}}(y), \ldots, \sup _{y \in\left(a_{n},+\infty\right)} \mu_{X_{n}}(y)\right\}\right\} \\
& =1 \text {. } \\
& R_{p}(t)=\sup _{u>t} \mu_{X}(u)=\sup \left(t, a_{n}\right] \cup\left(a_{n},+\infty\right) \mu_{X}(u) \\
& =\max \left\{\sup _{y \in\left(t, a_{n}\right]} \mu_{X_{n}}(y), \sup _{y \in\left(a_{n},+\infty\right)} \max \right. \\
& \left.\cdot\left\{\mu_{X_{1}}(y), \mu_{X_{2}}(y), \ldots, \mu_{X_{n}}(y)\right\}\right\} \\
& =1 \text {. } \\
& R_{p}(t)=\sup _{u>t} \mu_{X}(u) \\
& =\sup _{(t,+\infty)} \max \left\{\mu_{X_{1}}(y), \mu_{X_{2}}(y), \ldots, \mu_{X_{n}}(y)\right\} \\
& =\max \left\{\mu_{X_{1}}(t), \mu_{X_{2}}(t), \ldots, \mu_{X_{n}}(t)\right\} . \\
& R_{p}(t)=\sup _{u>t} \mu_{X}(u)= \begin{cases}1, & t \leq a_{n} \\
\max \left\{\mu_{X_{1}}(t), \mu_{X_{2}}(t), \ldots, \mu_{X_{n}}(t)\right\}, & t>a_{n} .\end{cases}
\end{aligned}
$$

When $t>a_{n}$, 
Remark 3. From the lifetime of the parallel system, $X=\max \left\{X_{1}, X_{2}, \ldots, X_{n}\right\}$, and equations (46) and (47), we can know that the property of $\mu_{X_{i}}(u)$ what we are focused on is the supremum value when $u \leq x$. For the series system case, due to the lifetime is defined by $X=\min \left\{X_{1}, X_{2}, \ldots, X_{n}\right\}$, the property of $\mu_{X_{i}}(u)$ what we are focused on is the supremum value when $u \geq x$. This is a big difference between the proof of the theory in series and parallel systems.

Specially, when the lifetime of the components are assumed as symmetrical Gaussian fuzzy variables, i.e., $L(x)$ and $R(x)$ have a normal distribution. We have the following corollary.

Corollary 2. Given a possibility space $\left(U, \Phi, P_{\text {oss }}\right)$ and a parallel system consisting of $n$ components, the possibility distribution function of the lifetime of components $X_{i}(i=$ $1,2, \ldots, n)$ are

$$
\mu_{X_{i}}(x)=\exp \left(-\left(\frac{x-m_{i}}{h}\right)^{2}\right),
$$

without loss of generality; we assume $m_{1} \leq m_{2} \leq \cdots \leq m_{n}$, then the possibility distribution function of the system lifetime $X$ is

$$
\mu_{X}(x)=\exp \left(-\left(\frac{x-m_{n}}{h}\right)^{2}\right)
$$

Moreover, the Posbist reliability of the parallel system is

$$
R_{p}(t)=\sup _{u>t} \mu_{X}(u)= \begin{cases}1, & t \leq m_{n} \\ \exp \left(-\left(\frac{t-m_{n}}{h}\right)^{2}\right), & t>m_{n} .\end{cases}
$$

Remark 4. Given a possibility space $\left(U, \Phi, P_{\text {oss }}\right)$ and a parallel system consisting of $n$ components, assume the possibility distribution function of the lifetime of the components $X_{i}(i=1,2, \ldots, n)$ are identically and independently distributed ones $\mu_{X_{1}}(x)$; then, there exists a real number $a$, such that $\mu_{X}(x)=\mu_{X_{1}}(x)$ and $R_{p}(t)=\sup _{u>t}$ $\mu_{X}(u)= \begin{cases}1, & t \leq a, \\ \mu_{X_{1}}(t), & t>a .\end{cases}$

3.3. Posbist Reliability of a Series-Parallel System. Given a possibility space $\left(U, \Phi, P_{\text {oss }}\right)$, we consider a series-parallel system consisting of $m$ systems, each system is a parallel system consisting of $n$ components, as shown in Figure 3. Suppose $X$ is the system lifetime, $X_{1}, X_{2}, \ldots, X_{m}$ are the lifetimes of $m$ parallel subsystems, and $X_{11}, X_{12}, \ldots, X_{1 n}, X_{21}, X_{22}, \ldots, X_{2 n}, \ldots, X_{m 1}, X_{m 2}, \ldots, X_{m n}$ , are the lifetimes of the $m n$ components, respectively. Then, the lifetime of system $X$ is $X=\min \{\max$ $\left\{X_{11}, X_{12}, \ldots, X_{1 n}\right\}, \max \left\{X_{21}, X_{22}, \ldots X_{2 n}\right\}, \ldots, \max \left\{X_{m 1}\right.$, $\left.\left.X_{m 2}, \ldots, X_{m n}\right\}\right\}=\min \left\{X_{1}, X_{2} \ldots, X_{m}\right\}$.

Theorem 3. Given a possibility space $\left(U, \Phi, P_{\text {oss }}\right)$ and a series-parallel system consisting of $m n$ components, the lifetime of components $X_{i j}(i=1,2, \ldots, m ; j=1,2, \ldots, n)$ are unrelated and each a normal, strictly convex fuzzy variable, with continuous possibility distribution functions $\mu_{X_{11}}(x), \mu_{X_{12}}(x), \ldots, \mu_{X_{1 n}}(x), \ldots, \mu_{X_{m n}}(x)$, respectively. Let the system lifetime be $X$ with possibility distribution function $\mu_{X}(x)$ and each parallel subsystem lifetime be $X_{i}$ with possibility distribution function $\mu_{X_{i}}(x)(i=1,2, \ldots, m)$. Then, there exist $m n$ positive numbers $a_{11}, a_{12}, \ldots, a_{1 n}$, $a_{21}, a_{22}, \ldots, a_{2 n}, \ldots, a_{m n}$ (without loss of generality, we assume $\quad a_{11} \leq a_{12} \leq \cdots \leq a_{1 n} ; \quad a_{21} \leq a_{22} \leq \cdots \leq a_{2 n} ; \quad \cdots$; $a_{m 1} \leq a_{m 2} \leq \cdots \leq a_{m n}$ and $\left.a_{1 n} \leq a_{2 n} \leq \cdots \leq a_{m n}\right)$, such that the possibility distribution function of $X$ is

$$
\mu_{X}(x)= \begin{cases}\max \left\{\mu_{X_{1}}(x), \mu_{X_{2}}(x), \ldots, \mu_{X_{m}}(x)\right\}, & x \leq a_{1 n}, \\ \mu_{X_{1}}(x), & a_{1 n}<x \leq a_{2 n}, \\ \min \left\{\mu_{X_{1}}(x), \mu_{X_{2}}(x)\right\}, & a_{2 n}<x \leq a_{3 n}, \\ \ldots & \\ \min \left\{\mu_{X_{1}}(x), \mu_{X_{2}}(x), \ldots, \mu_{X_{m-1}}(x)\right\}, & a_{(m-1) n}<x \leq a_{m n}, \\ \min \left\{\mu_{X_{1}}(x), \mu_{X_{2}}(x), \ldots, \mu_{X_{m}}(x)\right\}, & x>a_{m n},\end{cases}
$$

where

$$
\mu_{X_{i}}(x)=\left\{\begin{array}{l}
\min \left\{\mu_{X_{i 1}}(x), \mu_{X_{i 2}}(x), \ldots, \mu_{X_{i n}}(x)\right\}, x \leq a_{i 1}, \\
\min \left\{\mu_{X_{i 2}}(x), \mu_{X_{i 3}}(x), \ldots, \mu_{X_{i n}}(x)\right\}, a_{i 1}<x \leq a_{i 2}, \\
\min \left\{\mu_{X_{i 3}}(x), \mu_{X_{i 4}}(x), \ldots, \mu_{X_{i n}}(x)\right\}, a_{i 2}<x \leq a_{i 3} \\
\ldots \\
\mu_{X_{i n}}(x), a_{i(n-1)}<x \leq a_{i n}, \\
\max \left\{\mu_{X_{i 1}}(x), \mu_{X_{i 2}}(x), \ldots, \mu_{X_{i n}}(x)\right\}, x>a_{i n},
\end{array}\right.
$$

$(i=1,2, \ldots, m$,$) . Moreover, the Posbist reliability of the$ series-parallel system is 


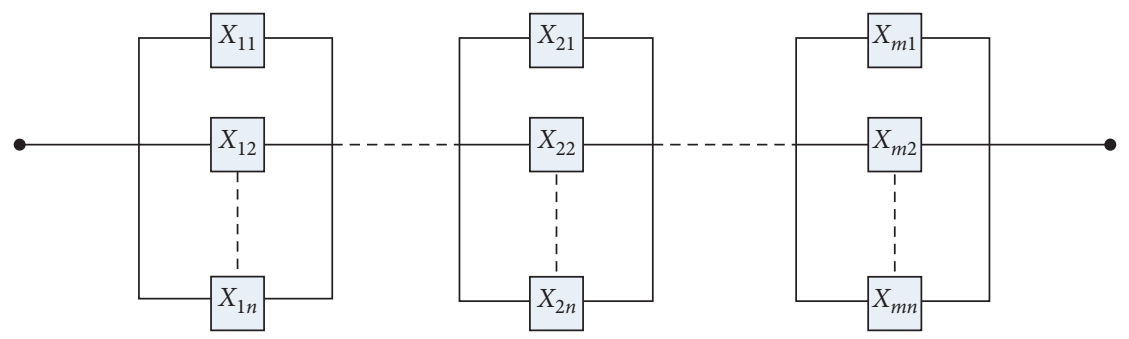

Figure 3: The logic block diagram of a series-parallel system.

$$
R_{s p}(t)=\sup _{u>t} \mu_{X}(u)=\left\{\begin{array}{l}
1, t \leq a_{1 n}, \\
\mu_{X_{1}}(t), a_{1 n}<t \leq a_{2 n}, \\
\min \left\{\mu_{X_{1}}(t), \mu_{X_{2}}(t)\right\}, a_{2 n}<t \leq a_{3 n}, \\
\ldots \\
\min \left\{\mu_{X_{1}}(t), \mu_{X_{2}}(t), \ldots, \mu_{X_{m-1}}(t)\right\}, a_{(m-1) n}<t \leq a_{m n} \\
\min \left\{\mu_{X_{1}}(t), \mu_{X_{2}}(t), \ldots, \mu_{X_{m}}(t)\right\}, t>a_{m n} .
\end{array}\right.
$$

Proof. The considered system can be regarded as a series system consisting of $m$ systems, each system is a parallel system. For each component $X_{i j}(i=1,2, \ldots, m ; j=1,2, \ldots, n)$, there exists a unique real number $a_{i j} \in R^{+}$, such that

$$
\sup _{u \leq x} \mu_{X_{i j}}(u)= \begin{cases}\mu_{X_{i j}}(x), & \text { if } x \leq a_{i j} \\ 1, & \text { if } x>a_{i j}\end{cases}
$$

According to Theorem 2, the possibility distribution function $\mu_{X_{i}}(x)(i=1,2, \ldots, m)$ of each parallel system is $\mu_{X_{i}}(x)=\left\{\begin{array}{l}\min \left\{\mu_{X_{i 1}}(x), \mu_{X_{i 2}}(x), \ldots, \mu_{X_{i n}}(x)\right\}, x \leq a_{i 1}, \\ \min \left\{\mu_{X_{i 2}}(x), \mu_{X_{i 3}}(x), \ldots, \mu_{X_{i n}}(x)\right\}, a_{i 1}<x \leq a_{i 2}, \\ \min \left\{\mu_{X_{i 3}}(x), \mu_{X_{i 4}}(x), \ldots, \mu_{X_{i n}}(x)\right\}, a_{i 2}<x \leq a_{i 3}, \\ \ldots \\ \mu_{X_{i n}}(x), a_{i(n-1))<x \leq a_{i n}, \max \left\{\mu_{X_{i 1}}(x), \mu_{X_{i 2}}(x), \ldots, \mu_{X_{i n}}(x)\right\}, x>a_{i n} .}\end{array}\right.$
For each possibility distribution function $\mu_{X_{i}}(x)$ and a real number $a_{i n}$, we have

$$
\sup _{u \geq x} \mu_{X_{i}}(u)= \begin{cases}1, & \text { if } x \leq a_{i n} \\ \mu_{X_{i}}(x), & \text { if } x>a_{i n}\end{cases}
$$

Based on Theorem 1, the possibility distribution function of $X$ is

$$
\mu_{X}(x)=\left\{\begin{array}{l}
\max \left\{\mu_{X_{1}}(x), \mu_{X_{2}}(x), \ldots, \mu_{X_{m}}(x)\right\}, x \leq a_{1 n}, \\
\mu_{X_{1}}(x), a_{1 n}<x \leq a_{2 n}, \\
\min \left\{\mu_{X_{1}}(x), \mu_{X_{2}}(x)\right\}, a_{2 n}<x \leq a_{3 n}, \\
\ldots \\
\min \left\{\mu_{X_{1}}(x), \mu_{X_{2}}(x), \ldots, \mu_{X_{m-1}}(x)\right\}, a_{(m-1) n}<x \leq a_{m n}, \\
\min \left\{\mu_{X_{1}}(x), \mu_{X_{2}}(x), \ldots, \mu_{X_{m}}(x)\right\}, x>a_{m n},
\end{array},\right.
$$

and the Posbist reliability of the series-parallel system is

$$
R_{s p}(t)=\sup _{u>t} \mu_{X}(u)=\left\{\begin{array}{l}
1, t \leq a_{1 n}, \\
\mu_{X_{1}}(t), a_{1 n}<t \leq a_{2 n} \\
\min \left\{\mu_{X_{1}}(t), \mu_{X_{2}}(t)\right\}, a_{2 n}<t \leq a_{3 n} \\
\ldots \\
\min \left\{\mu_{X_{1}}(t), \mu_{X_{2}}(t), \ldots, \mu_{X_{m-1}}(t)\right\}, a_{(m-1) n}<t \leq a_{m n} \\
\min \left\{\mu_{X_{1}}(t), \mu_{X_{2}}(t), \ldots, \mu_{X_{m}}(t)\right\}, t>a_{m n} .
\end{array}\right.
$$

Specially, when the lifetime of the components are assumed as symmetrical Gaussian fuzzy variables, i.e., $L(x)$ and $R(x)$ have a normal distribution. We have the following corollary. 
Corollary 3. Given a possibility space $\left(U, \Phi, P_{\text {oss }}\right)$ and a series-parallel system consisting of $m$ n components, the possibility distribution function of the lifetime of components $X_{i j}(i=1,2, \ldots, m ; j=1,2, \ldots, n)$ are

$$
\mu_{X_{i j}}(x)=\exp \left(-\left(\frac{x-\bar{m}_{i j}}{h}\right)^{2}\right) \text {. }
$$

Let $M_{1}=\max \left\{\bar{m}_{11}, \bar{m}_{12}, \ldots, \bar{m}_{1 n}\right\}, \quad M_{2}=\max \left\{\bar{m}_{21}\right.$, $\left.\bar{m}_{22}, \ldots, \bar{m}_{2 n}\right\}, \ldots, M_{m}=\max \left\{\bar{m}_{m 1}, \bar{m}_{m 2}, \ldots, \bar{m}_{m n}\right\}$, and $M=\min \left\{M_{1}, M_{2}, \ldots, M_{m}\right\}$; then, the possibility distribution function of the system lifetime $X$ is

$$
\mu_{X}(x)=\exp \left(-\left(\frac{x-M}{h}\right)^{2}\right) \text {. }
$$

Moreover, the Posbist reliability of the series-parallel system is

$$
R_{s p}(t)=\sup _{u>t} \mu_{X}(u)= \begin{cases}1, & t \leq M, \\ \exp \left(-\left(\frac{t-M}{h}\right)^{2}\right), & t>M .\end{cases}
$$

Remark 5. Given a possibility space $\left(U, \Phi, P_{\text {oss }}\right)$ and a seriesparallel system consisting of $m n$ components, assume the possibility distribution function of the lifetime of the components $X_{i j}(i=1,2, \ldots, m ; j=1,2, \ldots, n)$ are identically and independently distributed ones $\mu_{X_{11}}(x)$; then, there exists a real number $a$, such that $\mu_{X}(x)=\mu_{X_{11}}(x)$ and

$$
R_{s p}(t)=\sup _{u>t} \mu_{X}(u)= \begin{cases}1, & t \leq a, \\ \mu_{X_{11}}(t), & t>a .\end{cases}
$$

3.4. Posbist Reliability of a Parallel-Series System. Given a possibility space $\left(U, \Phi, P_{\text {oss }}\right)$, we consider a parallel-series system consisting of $m$ systems, each system is a series system consisting of $n$ components, as shown in Figure 4. Suppose $X$ is the system lifetime, $X_{1}, X_{2}, \ldots, X_{m}$ are the lifetimes of $m$ series systems and $X_{11}, X_{12}, \ldots, X_{1 n}, X_{21}, X_{22}$, $\ldots, X_{2 n}, \ldots, X_{m 1}, X_{m 2}, \ldots, X_{m n}$ are the lifetimes of the $m n$ components, respectively. Then, the lifetime of system $X$ is $X=\max \left\{\min \left\{X_{11}, X_{12}, \ldots, X_{1 n}\right\}, \min \left\{X_{21}, X_{22}\right.\right.$, $\left.\left.\ldots, X_{2 n}\right\}, \ldots, \min \left\{X_{m 1}, X_{m 2}, \ldots, X_{m n}\right\}\right\}=\max \left\{X_{1}\right.$, $\left.X_{2}, \ldots, X_{m}\right\}$.

Theorem 4. Given a possibility space $\left(U, \Phi, P_{\text {oss }}\right)$ and a parallel-series system consisting of $m n$ components, the lifetime of components $X_{i j}(i=1,2, \ldots, m ; j=1,2, \ldots, n)$ are unrelated and each a normal, strictly convex fuzzy variable,

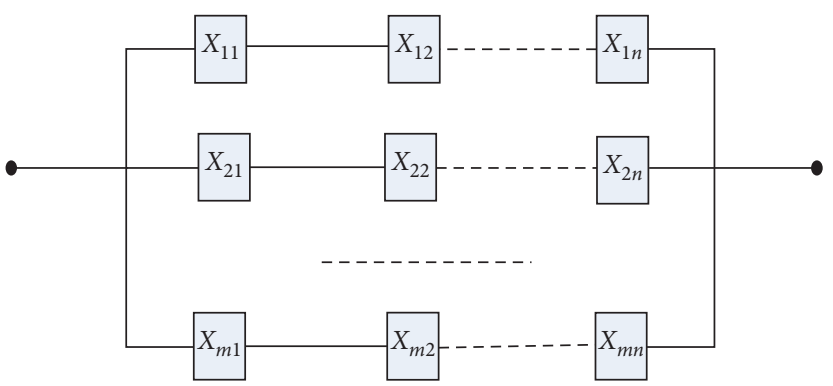

FIgURE 4: The logic block diagram of a parallel-series system.

with continuous possibility distribution functions $\mu_{X_{11}}(x), \mu_{X_{12}}(x), \ldots, \mu_{X_{1 n}}(x), \ldots, \mu_{X_{m n}}(x)$, respectively. Let the system lifetime be $X$ with possibility distribution function $\mu_{X}(x)$ and each series system lifetime be $X_{i}$ with possibility distribution function $\mu_{X_{i}}(x)(i=1,2, \ldots, m)$. Then, there exist $m n$ positive numbers $a_{11}, a_{12}, \ldots, a_{1 n}, a_{21}, a_{22}, \ldots, a_{2 n}, \ldots, a_{m n}$ (without loss of generality, we assume $a_{11} \leq a_{12} \leq \cdots \leq a_{1 n}$; $a_{21} \leq a_{22} \leq \cdots \leq a_{2 n} ; \quad \ldots ; \quad a_{m 1} \leq a_{m 2} \leq \cdots \leq a_{m n} \quad$ and $\left.a_{11} \leq a_{21} \leq \cdots \leq a_{m 1}\right)$, such that the possibility distribution function of $X$ is

$$
\mu_{X}(x)=\left\{\begin{array}{l}
\min \left\{\mu_{X_{1}}(x), \mu_{X_{2}}(x), \ldots, \mu_{X_{m}}(x)\right\}, x \leq a_{11}, \\
\min \left\{\mu_{X_{2}}(x), \mu_{X_{3}}(x), \ldots, \mu_{X_{m}}(x)\right\}, a_{11}<x \leq a_{21}, \\
\min \left\{\mu_{X_{3}}(x), \mu_{X_{4}}(x), \ldots, \mu_{X_{m}}(x)\right\}, a_{21}<x \leq a_{31}, \\
\ldots \\
\mu_{X_{m}}(x), a_{(m-1) 1}<x \leq a_{m 1}, \\
\max \left\{\mu_{X_{1}}(x), \mu_{X_{2}}(x), \ldots, \mu_{X_{m}}(x)\right\}, x>a_{m 1},
\end{array}\right.
$$

where

$$
\mu_{X_{i}}(x)=\left\{\begin{array}{l}
\max \left\{\mu_{X_{i 1}}(x), \mu_{X_{i 2}}(x), \ldots, \mu_{X_{i n}}(x)\right\}, x \leq a_{i 1}, \\
\mu_{X_{i 1}}(x), a_{i 1}<x \leq a_{i 2}, \\
\min \left\{\mu_{X_{i 1}}(x), \mu_{X_{i 2}}(x)\right\}, a_{i 2}<x \leq a_{i 3}, \\
\cdots \\
\min \left\{\mu_{X_{i 1}}(x), \mu_{X_{i 2}}(x), \ldots, \mu_{X_{i(n-1)}}(x)\right\}, a_{(i-n)}<x \leq a_{i n}, \\
\min \left\{\mu_{X_{i 1}}(x), \mu_{X_{i 2}}(x), \ldots, \mu_{X_{i n}}(x)\right\}, x>a_{i n},
\end{array}\right.
$$

$(i=1,2, \ldots, m)$

Moreover, the Posbist reliability of the parallel-series system is

$$
R_{p s}(t)=\sup _{u>t} \mu_{X}(u)= \begin{cases}1, & t \leq a_{m 1} \\ \max \left\{\mu_{X_{1}}(t), \mu_{X_{2}}(t), \ldots, \mu_{X_{m}}(t)\right\}, & t>a_{m 1}\end{cases}
$$


Proof. The considered system can be regarded as a parallel system consisting of $m$ systems, each system is a series system. For each component $X_{i j}(i=1,2, \ldots, m ; j=1,2, \ldots, n)$, there exists a unique real number $a_{i j} \in R^{+}$, such that

$$
\sup _{u \geq x} \mu_{X_{i j}}(u)= \begin{cases}1, & \text { if } x \leq a_{i j} \\ \mu_{X_{i j}}(x), & \text { if } x>a_{i j}\end{cases}
$$

According to Theorem 1, the possibility distribution function $\mu_{X_{i}}(x)(i=1,2, \ldots, m)$ of each series system is

$$
\mu_{X_{i}}(x)=\left\{\begin{array}{l}
\max \left\{\mu_{X_{i 1}}(x), \mu_{X_{i 2}}(x), \ldots, \mu_{X_{i n}}(x)\right\}, x \leq a_{i 1}, \\
\mu_{X_{i 1}}(x), a_{i 1}<x \leq a_{i 2}, \\
\min \left\{\mu_{X_{i 1}}(x), \mu_{X_{i 2}}(x)\right\}, a_{i 2}<x \leq a_{i 3}, \\
\ldots \\
\min \left\{\mu_{X_{i 1}}(x), \mu_{X_{i 2}}(x), \ldots, \mu_{X_{i(n-1)}}(x)\right\}, a_{(i-1) n}<x \leq a_{i n}, \\
\min \left\{\mu_{X_{i 1}}(x), \mu_{X_{i 2}}(x), \ldots, \mu_{X_{i n}}(x)\right\}, x>a_{i n} .
\end{array}\right.
$$

For each possibility distribution function $\mu_{X_{i}}(x)$ and a real number $a_{i 1}$, we have

$$
\sup _{u \leq x} \mu_{X_{i}}(u)= \begin{cases}\mu_{X_{i}}(x), & \text { if } x \leq a_{i 1} \\ 1, & \text { if } x>a_{i 1}\end{cases}
$$

Based on Theorem 2, the possibility distribution function of $X$ is

$$
\mu_{X}(x)=\left\{\begin{array}{l}
\min \left\{\mu_{X_{1}}(x), \mu_{X_{2}}(x), \ldots, \mu_{X_{m}}(x)\right\}, x \leq a_{11}, \\
\min \left\{\mu_{X_{2}}(x), \mu_{X_{3}}(x), \ldots, \mu_{X_{m}}(x)\right\}, a_{11}<x \leq a_{21}, \\
\min \left\{\mu_{X_{3}}(x), \mu_{X_{4}}(x), \ldots, \mu_{X_{m}}(x)\right\}, a_{21}<x \leq a_{31}, \\
\ldots \\
\mu_{X_{m}}(x), a_{(m-1) 1}<x \leq a_{m 1}, \\
\max \left\{\mu_{X_{1}}(x), \mu_{X_{2}}(x), \ldots, \mu_{X_{m}}(x)\right\}, x>a_{m 1},
\end{array}\right.
$$

and the Posbist reliability of the parallel-series system is

$$
R_{p s}(t)=\sup _{u>t} \mu_{X}(u)= \begin{cases}1, & t \leq a_{m 1}, \\ \max \left\{\mu_{X_{1}}(t), \mu_{X_{2}}(t), \ldots, \mu_{X_{m}}(t)\right\}, & t>a_{m 1} .\end{cases}
$$

Specially, when the lifetime of components are assumed as symmetrical Gaussian fuzzy variables, i.e., $L(x)$ and $R(x)$ are selected as the normal shape. We have the following corollary.

Corollary 4. Given a possibility space $\left(U, \Phi, P_{\text {oss }}\right)$ and a parallel-series system consisting of $m n$ components, the possibility distribution function of the lifetime of components $X_{i j}(i=1,2, \ldots, m ; j=1,2, \ldots, n)$ are

$$
\mu_{X_{i j}}(x)=\exp \left(-\left(\frac{x-\bar{m}_{i j}}{h}\right)^{2}\right) \text {. }
$$

Let $\quad \widehat{M}_{1}=\min \left\{\bar{m}_{11}, \bar{m}_{12}, \ldots, \bar{m}_{1 n}\right\}, \quad \widehat{M}_{2}=\min \left\{\bar{m}_{21}\right.$, $\left.\bar{m}_{22}, \ldots, \bar{m}_{2 n}\right\}, \ldots, \widehat{M}_{m}=\min \left\{\bar{m}_{m 1}, \bar{m}_{m 2}, \ldots, \bar{m}_{m n}\right\}$ and $\widehat{M}=\max \left\{M_{1}, M_{2}, \ldots, M_{m}\right\}$; then, the possibility distribution function of the system lifetime $X$ is

$$
\mu_{X}(x)=\exp \left(-\left(\frac{x-\widehat{M}}{h}\right)^{2}\right) \text {. }
$$

Moreover, the Posbist reliability of the parallel-series system is

$$
R_{p s}(t)=\sup _{u>t} \mu_{X}(u)= \begin{cases}1, & t \leq \hat{M}, \\ \exp \left(-\left(\frac{t-\widehat{M}}{h}\right)^{2}\right), & t>\widehat{M} .\end{cases}
$$

Remark 6. Given a possibility space $\left(U, \Phi, P_{\text {oss }}\right)$ and a parallel-series system consisting of $m n$ components, assume the possibility distribution function of the lifetime of components $X_{i j}(i=1,2, \ldots, m ; j=1,2, \ldots, n)$ are identically and independently distributed ones $\mu_{X_{1}}(x)$; then, there exists a real number $a$, such that $\mu_{X}(x)=\mu_{X_{11}}(x)$ and $R_{p s}(t)=\sup _{u>t} \mu_{X}(u)=\left\{\begin{array}{ll}1, & t \leq a \\ \mu_{X_{11}}(t), & t>a .\end{array}\right.$.

Remark 7. Assume that the components of each system are identically and independently distributed ones and components between each system are different; combining $\mathrm{Re}$ marks 2, 4, 5, and 6, one can obtain that the Posbist reliability of each system is independent of the number of components. Moreover, if components between each system are also assumed to be the same, the Posbist reliability of each system is the same.

\section{Numerical Examples}

4.1. The Posbist Reliability of the Typical System. We are in a position to compare the Posbist reliability of a series system, parallel system, series-parallel system, and parallel-series system. Each system is consisting of four components. The chosen parameter values are $m_{1(11)}=140, m_{2(12)}=160$, $m_{3(21)}=180, \quad m_{4(22)}=200, \quad h_{1(11)}=50, \quad h_{2(12)}=70$, $h_{3(21)}=90$, and $h_{4(22)}=110$. The possibility distribution function of the lifetime of each component is

$$
\begin{aligned}
& \mu_{X_{1(11)}}(x)=\exp \left(-\left(\frac{x-140}{50}\right)^{2}\right), \\
& \mu_{X_{2(12)}}(x)=\exp \left(-\left(\frac{x-160}{70}\right)^{2}\right), \\
& \mu_{X_{3(21)}}(x)=\exp \left(-\left(\frac{x-180}{90}\right)^{2}\right), \\
& \mu_{X_{4(22)}}(x)=\exp \left(-\left(\frac{x-200}{110}\right)^{2}\right) .
\end{aligned}
$$


When the considered systems are series and parallel system, the lifetime of components are $X_{1}, X_{2}, X_{3}$, and $X_{4}$ with continuous possibility distribution functions $\mu_{X_{1}}(x)$, $\mu_{X_{2}}(x), \mu_{X_{3}}(x)$, and $\mu_{X_{4}}(x)$. When the considered systems are series-parallel and parallel-series system, the lifetime of the components are $X_{11}, X_{12}, X_{21}$, and $X_{22}$ with continuous possibility distribution functions $\mu_{X_{11}}(x), \mu_{X_{12}}(x), \mu_{X_{21}}(x)$, and $\mu_{X_{22}}(x)$. From (89), we have $\mu_{X_{1}}(x)=\mu_{X_{11}}(x)$, $\mu_{X_{2}}(x)=\mu_{X_{12}}(x), \mu_{X_{3}}(x)=\mu_{X_{21}}(x)$, and $\mu_{X_{4}}(x)=\mu_{X_{22}}(x)$. Let $t=170$ (hour), the Posbist reliability of each system is calculated as follows.

(1) The series system: from (9), the Posbist reliability for the series system consisting of four components is

$$
R_{s}(t)=\left\{\begin{array}{l}
1, t \leq 140, \\
\exp \left(-\left(\frac{x-140}{50}\right)^{2}\right), 140<t \leq 160 \\
\min \left\{\exp \left(-\left(\frac{x-140}{50}\right)^{2}\right), \exp \left(-\left(\frac{x-160}{70}\right)^{2}\right)\right\}, 160<t \leq 180, \\
\min \left\{\exp \left(-\left(\frac{x-140}{50}\right)^{2}\right), \exp \left(-\left(\frac{x-160}{70}\right)^{2}\right), \exp \left(-\left(\frac{x-180}{90}\right)^{2}\right)\right\}, 180<t \leq 200, \\
\min \left\{\exp \left(-\left(\frac{x-140}{50}\right)^{2}\right), \exp \left(-\left(\frac{x-160}{70}\right)^{2}\right), \exp \left(-\left(\frac{x-180}{90}\right)^{2}\right), \exp \left(-\frac{x-200^{2}}{110}\right)\right\}, t>200 .
\end{array}\right.
$$

When $t=170$ (hour), we can obtain

$R_{s}(170)=\min \left\{\exp \left(-\left(\frac{170-140}{50}\right)^{2}\right), \exp \left(-\left(\frac{170-160}{70}\right)^{2}\right)\right\}$

$=0.5488$.
(2) The parallel system: from (43), the Posbist reliability for the parallel system consisting of four components is

$$
R_{p}(t)=\left\{\begin{array}{l}
1, t \leq 200 \\
\max \left\{\exp \left(-\left(\frac{x-140}{50}\right)^{2}\right), \exp \left(-\frac{x-160^{2}}{70}\right), \exp \left(-\left(\frac{x-180}{90}\right)^{2}\right), \exp \left(-\left(\frac{x-200}{110}\right)^{2}\right)\right\}, t>200 .
\end{array}\right.
$$

When $t=170$ (hour), we can obtain

$$
\mu_{X_{1}}(x)=\left\{\begin{array}{l}
\min \left\{\exp \left(-\left(\frac{140-x}{50}\right)^{2}\right), \exp \left(-\left(\frac{160-x}{70}\right)^{2}\right)\right\}, x \leq 140, \\
\exp \left(-\left(\frac{160-x}{70}\right)^{2}\right), 140<x \leq 160, \\
\max \left\{\exp \left(-\left(\frac{x-140}{50}\right)^{2}\right), \exp \left(-\left(\frac{x-160}{70}\right)^{2}\right)\right\}, x>160,
\end{array}\right.
$$

reliability for the series-parallel system consisting of four components is

$$
R_{s p}(t)=\left\{\begin{array}{l}
1, t \leq 160 \\
\mu_{X_{1}}(t), 160<t \leq 200 \\
\min \left\{\mu_{X_{1}}(t), \mu_{X_{2}}(t)\right\}, t>200
\end{array}\right.
$$

$$
\mu_{X_{2}}(x)=\left\{\begin{array}{l}
\min \left\{\exp \left(-\left(\frac{180-x}{90}\right)^{2}\right), \exp \left(-\left(\frac{200-x}{110}\right)^{2}\right)\right\}, x \leq 180, \\
\exp \left(-\left(\frac{200-x}{110}\right)^{2}\right), 180<x \leq 200, \\
\max \left\{\exp \left(-\left(\frac{x-180}{90}\right)^{2}\right), \exp \left(-\left(\frac{x-200}{110}\right)^{2}\right)\right\}, x>200 .
\end{array}\right.
$$


When $t=170$ (hour), we can obtain

$$
\begin{aligned}
R_{s p}(170) & =\max \left\{\exp \left(-\left(\frac{170-140}{50}\right)^{2}\right) \exp \left(-\left(\frac{170-160}{70}\right)^{2}\right)\right\} \\
& =0.8669 .
\end{aligned}
$$

(4) The parallel-series system: from (80), the Posbist reliability for the parallel-series system consisting of four components is

$$
R_{p s}(t)= \begin{cases}1, & t \leq 180, \\ \max \left\{\mu_{X_{1}}(t), \mu_{X_{2}}(t)\right\}, & t>180,\end{cases}
$$

where

$$
\begin{aligned}
& \mu_{X_{1}}(x)=\left\{\begin{array}{l}
\max \left\{\exp \left(-\left(\frac{140-x}{50}\right)^{2}\right), \exp \left(-\left(\frac{160-x}{70}\right)^{2}\right)\right\}, x \leq 140, \\
\exp \left(-\left(\frac{x-140}{50}\right)^{2}\right), 140<x \leq 160, \\
\min \left\{\exp \left(-\left(\frac{x-140}{50}\right)^{2}\right), \exp \left(-\left(\frac{160-x}{70}\right)^{2}\right)\right\}, x>160,
\end{array}\right. \\
& \mu_{X_{2}}(x)=\left\{\begin{array}{l}
\max \left\{\exp \left(-\left(\frac{180-x}{90}\right)\right)^{2}, \exp \left(-\left(\frac{200-x}{110}\right)^{2}\right)\right\}, x \leq 180, \\
\exp \left(-\left(\frac{x-180}{90}\right)^{2}\right), 180<x \leq 200, \\
\min \left\{\exp \left(-\left(\frac{x-180}{90}\right)^{2}\right), \exp \left(-\left(\frac{x-200}{110}\right)^{2}\right)\right\}, x>200 .
\end{array}\right.
\end{aligned}
$$

When $t=170$ (hour), we can obtain

$$
R_{p s}(170)=1 \text {. }
$$

When $t=170$ (hour), the Posbist reliability for each system is shown in Table 1.The Posbist reliability for different systems consisting of four components at various points is shown in Figure 5.

From Table 1 and Figure 5, we have, at the same instant, for different systems consisting of the same components, the Posbist reliability of the parallel system is no less than the Posbist reliability of the parallel-series system. The Posbist reliability of the parallel-series system is no less than the Posbist reliability of the series-parallel system. The Posbist reliability of the series-parallel system is no less than the Posbist reliability of the series system.

4.2. A Comparison of Posbist and Probabilistic Reliability Models for Safety Evaluations. For the systems consisting of same components, at the same instant, safety evaluation is different between Posbist and probabilistic reliability models. $F(\cdot)$ represents the failure event of system; $p$ and $\pi$ represent the failure probability and failure possibility, respectively. We consider the series system, parallel system, series-parallel
TABle 1: The Posbist reliability for different systems of four components.

\begin{tabular}{lcccc}
\hline & Series & Parallel & Series-parallel & Parallel-series \\
\hline $\begin{array}{l}\text { Posbist } \\
\text { reliability }\end{array}$ & 0.5488 & 1 & 0.8669 & 1 \\
\hline
\end{tabular}

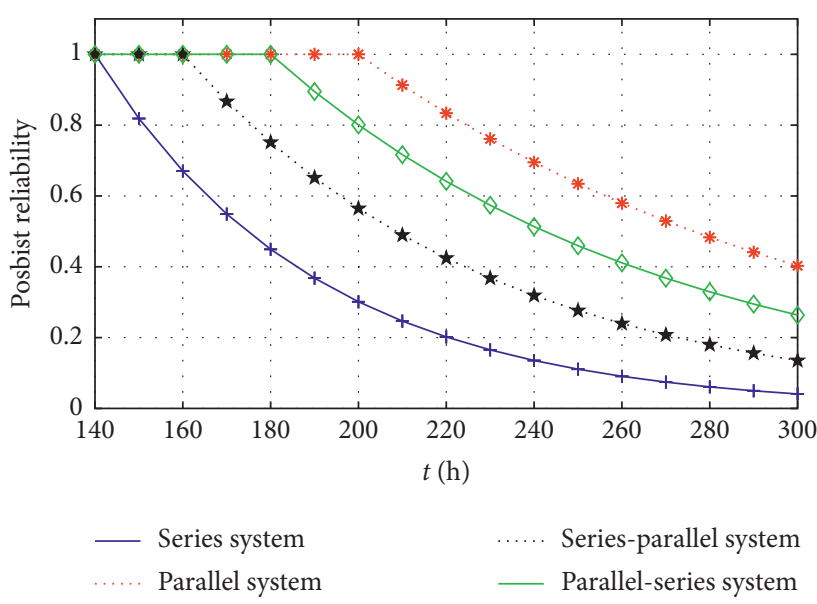

Figure 5: The Posbist reliability for different systems at various points (hours).

system, and parallel-series system consisting of $m n$ components. For the series-parallel system, each parallel system consists of $n$ components. For the parallel-series system, each series system consists of $n$ components. Assume the components are identically and independently distributed ones. For the series system, $F_{s}(p)=1-(1-p)^{m n}$ and $F_{s}(\pi)=\pi$. For the parallel system, $F_{p}(p)=p^{m n}$ and $F_{p}(\pi)=\pi$. For the series-parallel system, $F_{s p}(p)=1-\left(1-p^{n}\right)^{m}$ and $F_{s p}(\pi)=\pi$. For the parallel-series system, $F_{p s}(p)=\left(1-(1-p)^{n}\right)^{m}$ and $F_{p s}(\pi)=\pi$. Therefore, let $p=0.6$ and $\pi=0.5$, and the safety evaluation of each system is shown in Figure 6.

From Figure 6, for each system, the possibility of failure $F(\pi)$ is a constant. However, the probability of failure $F(p)$ changes. For the series system, as shown in (a) of Figure 6, the probability of failure $F(p)$ increases with the increasement of the number of components $m n$. For the parallel system, as shown in (b) of Figure 6, the probability of failure $F(p)$ decreases with the increasement of the number of components $m n$. For the series-parallel system, as shown in (c) of Figure 6, if the system components' number $n$ is fixed, the probability of failure $F(p)$ increases with the increasement of the number of $m$; if the number $m$ is fixed, the probability of failure $F(p)$ decreases with the increasement of the system components number of $n$. For the parallelseries system, as shown in (d) of Figure 6, if the system components' number $n$ is fixed, the probability of failure $F(p)$ decreases with the increasement of the number of $m$; if the number $m$ is fixed, the probability of failure $F(p)$ increases with the increasement of the system components number of $n$. Therefore, for different cases, a suitable model should be selected [27]. 


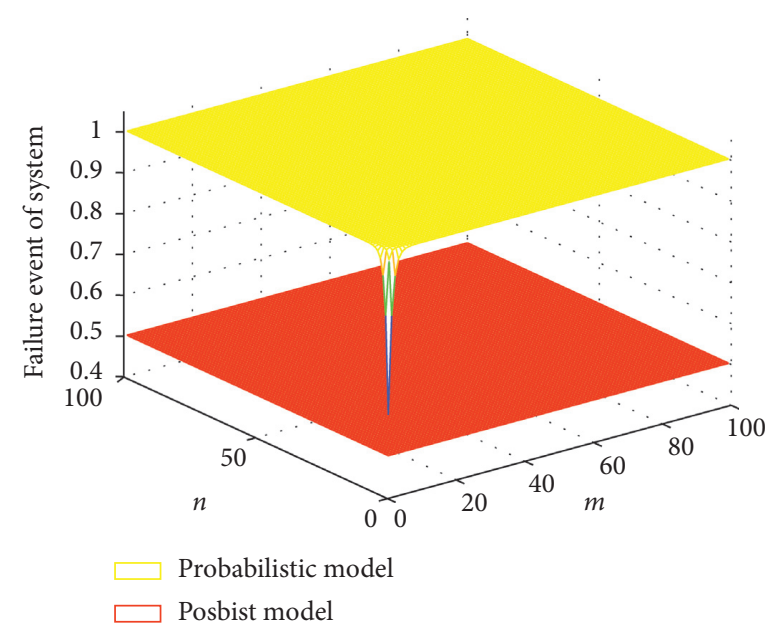

(a)

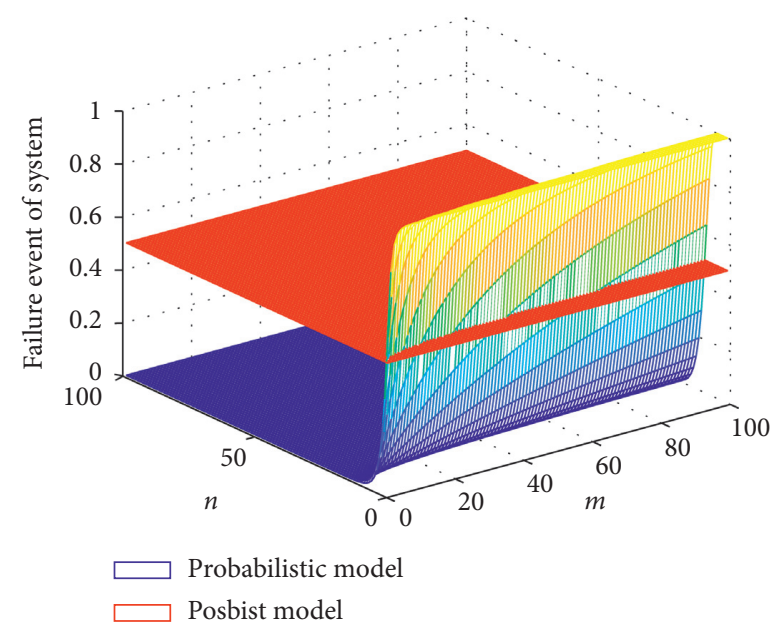

(c)

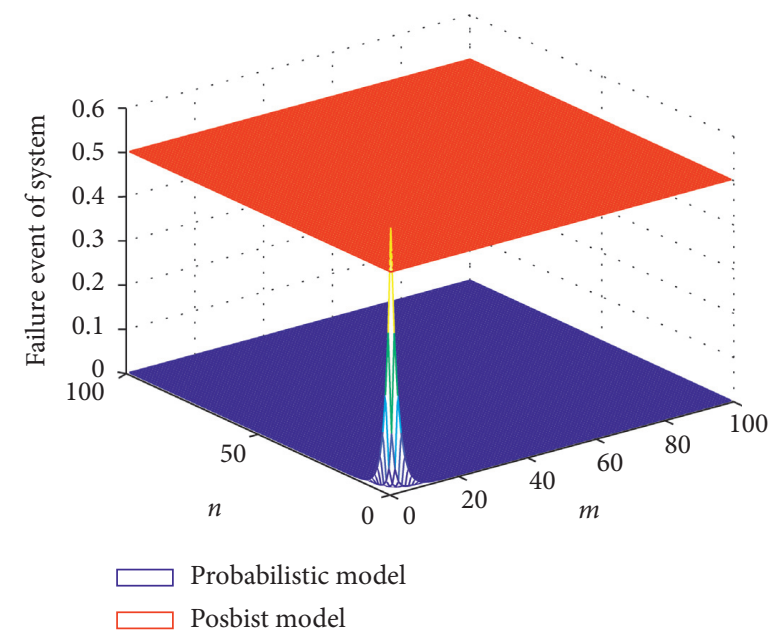

(b)

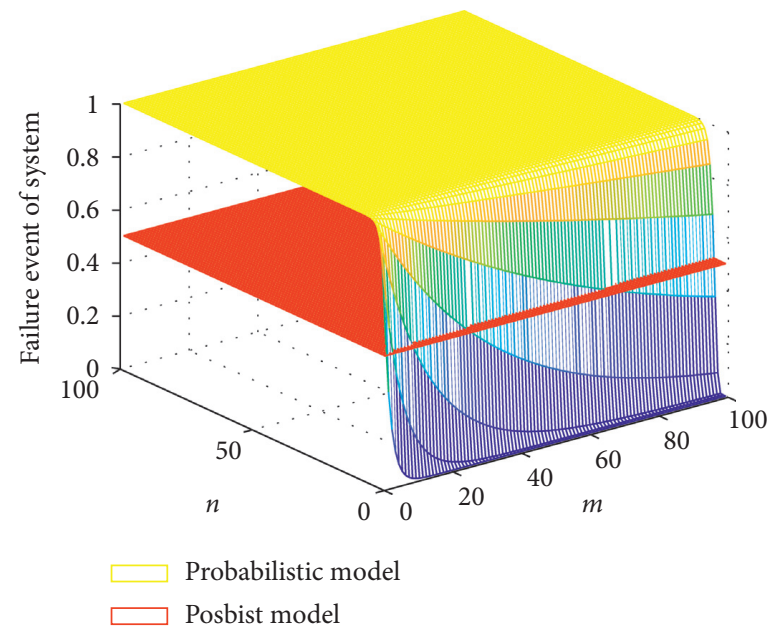

(d)

Figure 6: A comparison of Posbist and probabilistic reliability models for safety evaluations.

\section{Conclusion}

The problem of Posbist reliability theory for typical systems with multiple different components has been further investigated via the possibility theory. The results have been given when typical systems with $n$ different components. Moreover, our results have been concretely derived where the exactly value can be obtained at any moment, unlike existing ones only given by a lower and upper bound. Finally, numerical examples have been provided to compare the Posbist reliability between typical systems of four types: series, parallel, series-parallel, and parallel-series. Moreover, numerical safety evaluations have been given based on possibilistic and probabilistic models.

\section{Data Availability}

The data used to support the findings of this study are included within the article.

\section{Conflicts of Interest}

The authors declare that they have no conflicts of interest.

\section{Acknowledgments}

This work was supported by Opening Fund of Geomathematics Key Laboratory of Sichuan Province (scsxdz2019yb03).

\section{References}

[1] Z. Z. Mou and W. Y. Zhu, Mechanical Reliability Design, China Machine Press, Beijing, China, 1993.

[2] S. S. Gao and L. X. Zhang, Reliability Theory and Engineering Application, National Defense Industry Press, Beijing, China, 2002.

[3] H. Q. Li and G. Li, System Reliability Analysis and Design, Science Press, Beijing, China, 2003.

[4] G. Muscolino, R. Santoro, and A. Sofi, "Reliability analysis of structures with interval uncertainties under stationary stochastic excitations," Computer Methods in Applied Mechanics and Engineering, vol. 300, pp. 47-69, 2016.

[5] Y. G. Dong, Mechanical Fuzzy Reliability Design, China Machine Press, Beijing, China, 2000.

[6] H. Z. Huang, Fuzzy Design, China Machine Press, Beijing, China, 1999 
[7] I. Elishakoff, "Essay on uncertainties in elastic and viscoelastic structures: from A. M. Freudenthal's criticisms to modern convex modeling," Computers \& Structures, vol. 56, no. 6, pp. 871-895, 1995.

[8] A. Kaufmann, Introduction to the Fuzzy Subsets, Academic Press, New York, NY, USA, 1975.

[9] L. A. Zadeh, "Fuzzy sets as a basis for a theory of possibility," Fuzzy Sets and Systems, vol. 1, no. 1, pp. 3-28, 1978.

[10] T. Onisawa, "An application of fuzzy concepts to modelling of reliability analysis," Fuzzy Sets and Systems, vol. 37, no. 3, pp. 267-286, 1990.

[11] K. Y. Cai, C. Y. Wen, and M. L. Zhang, "Fuzzy variables as a basis for a theory of fuzzy reliability in the possibility context," Fuzzy Sets and Systems, vol. 42, no. 2, pp. 145-172, 1991.

[12] K. Y. Cai, C. Y. Wen, and M. L. Zhang, "Posbist reliability behavior of typical systems with two types of failure," Fuzzy Sets and Systems, vol. 43, no. 1, pp. 17-32, 1991.

[13] L. V. Utkin, "Fuzzy reliability of repairable systems in the possibility context," Microelectronics Reliability, vol. 34, no. 12, pp. 1865-1876, 1994.

[14] K.-Y. Cai, C.-Y. Wen, and M.-L. Zhang, "Fuzzy states as a basis for a theory of fuzzy reliability," Microelectronics Reliability, vol. 33, no. 15, pp. 2253-2263, 1993.

[15] K. Y. Cai, C. Y. Wen, and M. L. Zhang, "Mixture models in profust reliability theory," Microelectronics and Reliability, vol. 33, no. 6, pp. 985-993, 1995.

[16] V. Cutello, J. Montero, and J. Yáñez, "Structure functions with fuzzy states," Fuzzy Sets and Systems, vol. 83, no. 2, pp. 189-202, 1996.

[17] K.-Y. Cai, "Q-scale measures of fuzzy sets," Fuzzy Sets and Systems, vol. 66, no. 1, pp. 59-81, 1994.

[18] G. C. Marano and G. Quaranta, "A new possibilistic reliability index definition," Acta Mechanica, vol. 210, no. 3-4, pp. 291-303, 2010.

[19] H. Z. Huang, "Structural reliability analysis using fuzzy sets theory," Eksploatacja I Niezawodnosc-Maintenance and Reliability, vol. 14, pp. 284-294, 2012.

[20] Z. Wang, H.-Z. Huang, Y. Li, Y. Pang, and N.-C. Xiao, “An approach to system reliability analysis with fuzzy random variables," Mechanism and Machine Theory, vol. 52, pp. 3546, 2012.

[21] L. Zhang, J. Zhang, H. Zhai, and S. Zhou, “A new assessment method of mechanism reliability based on chance measure under fuzzy and random uncertainties," Eksploatacja I Niezawodnosc-Maintenance and Reliability, vol. 20, no. 2, pp. 219-228, 2018.

[22] L. P. He, The Reliability Analysis and Evaluation of Mechanical Systems Based on Possibilistic Measurements, Ph.D. Thesis, Dalian University of Technology, Liaoning, China, 2010.

[23] L. P. He, J. Xiao, H. Z. Huang, and Z. Q. Luo, "System reliability modeling and analysis in the possibility context," in Proceedings of the 2012 International Conference on Quality, Reliability, Risk, Maintenance, and Safety Engineering (ICQR2MSE), pp. 361-367, Chengdu, China, June 2012.

[24] Z. Li and K. C. Kapur, "Some perspectives to define and model reliability using fuzzy sets," Quality Engineering, vol. 25, no. 2, pp. 136-150, 2013.

[25] K. Y. Cai, Introduction to Fuzzy Reliability, Kluwer Academic Publishers, Boston, MA, USA, 1996.

[26] J. J. Ren, Y. P. Zhang, Y. H. Wei, C. F. Zhang, and B. Qin, "Reliability modeling and analysis for typical system with multi-components via possibility theory," in Proceeding of the 2015 International Conference on Electrical, Computer
Engineering and Electronics (ICECEE 2015), pp. 414-417, Jinan, China, May 2015.

[27] G. J. Klir, Fuzzy Sets: An Overview of Fundamentals, Applications and Personal Views, Beijing Normal University Press, Beijing, China, 2000. 